\title{
1 Identification of the pathway of Rhodoquinone biosynthesis in C.elegans
}

2

3 Samantha Del Borrello ${ }^{1 *}$, Margot Lautens ${ }^{1 *}$, Kathleen Dolan ${ }^{1}$, June H. Tan ${ }^{1}$, Mark A.

$4 \quad$ Spensley ${ }^{1,2}$, Amy A. Caudy ${ }^{1} \dagger$ and Andrew G. Fraser ${ }^{1} \dagger$

5

6

7

$8 * *=$ these authors contributed equally

10 Affiliation:

$11{ }^{1}$ The Donnelly Centre, University of Toronto, 160 College Street, Toronto M5S3E1.

12 2Phenalysys Inc. 50 Cambridge Avenue, Toronto, Ontario. M4K 2L3

13

14 † corresponding authors:

15 amy.caudy@utoronto.ca

16 andy.fraser@utoronto.ca 
Abstract

Parasitic helminths infect over a billion humans. To survive in the low oxygen environment of their hosts, these parasites use unusual anaerobic metabolism. This requires Rhodoquinone (RQ), an electron carrier that is made by very few animal species crucially it is not present in any parasitic hosts. RQ synthesis is thus an ideal target for anthelmintics but little is known about how $R Q$ is made and no drugs are known to block RQ synthesis. C.elegans makes RQ and can use RQ-dependent metabolic pathways - here, we use C.elegans genetics to identify the pathway for $R Q$ synthesis and show that C.elegans requires $R Q$ for survival in hypoxic conditions. Finally, we establish a robust assay for drugs that block RQ-dependent metabolism. This study identifies for the first time how RQ is made in any animal and establishes a novel assay that can drive the development of a new class of anthelmintic drugs.

\section{Introduction}

Soil-transmitted Helminths (STHs) are major human pathogens (World Health Organization, 2002). Over a billion humans are infected with an STH - the roundworm Ascaris lumbricoides, the whipworm Trichuris trichuria, and the hookworm Necator americanus account for most of these infections (World Health Organization, 2002). STHs are transmitted from human to human via the soil where eggs from human faeces develop into infective stages which then enter new hosts (reviewed in Brooker et al., 2006). On infection, STHs encounter a very different environment and require multiple strategies to be able to survive. One of the major changes is the availability of oxygen - while there is abundant oxygen outside their hosts, in many host tissues there is little available oxygen and the parasites must switch from aerobic respiration to anaerobic respiration. Crucially, the anaerobic metabolic pathways that STHs depend on while in their hosts are unusual and are not used in any host (Klockiewicz et al., 2002). Inhibiting these anaerobic pathways thus provides a way to attack the parasites while leaving the host unaffected.

During aerobic respiration in helminths, the great majority of ATP is made in the mitochondrion (Tielens, 1994; Tielens et al., 1984). Electrons enter the Electron Transport Chain (ETC) either at Complex I or via several quinone-coupled dehydrogenases (QDHs from here on). These QDHs include Succinate Dehydrogenase (Complex II) and Electron-Transferring Flavoprotein Dehydrogenase (ETFDH) (Komuniecki et al., 1989; Ma et al., 1993; Rioux and Komuniecki, 1984). The electrons entering the ETC are first transferred to the lipid soluble electron carrier Ubiquinone (UQ) (Crane et al., 1957; Mitchell, 1975). From UQ, they are ultimately carried to Complex III then IV where they are finally transferred onto oxygen as the terminal electron acceptor (see Fig 1a). Electron transport is coupled to proton pumping into the inner membrane space of the mitochondrion - this establishes a proton gradient which is used to power the F0F1-ATP synthase (Mitchell, 1961). When there is insufficient oxygen to accept electrons at Complex IV, or when inhibitors of Complex IV such as cyanide (Antonini et al., 1971; Nicholls

58 et al., 1972) are present, almost all animals stop using the ETC and rely on anaerobic glycolysis

59 to make ATP, generating lactate (Isom et al., 1975; Meyerhof, 1927). STHs, however, have

60 evolved a different solution that allow them to survive months in the hypoxic host environment. 
61 Electrons still enter the ETC at Complex I, Complex I still pumps protons to generate the proton motive force (PMF), and ATP is still made by the F0F1ATPase, powered by the PMF. However, rather than the electrons passing through the ETC to oxygen as the terminal electron acceptor, they exit the ETC immediately downstream of Complex I onto a number of alternative terminal electron acceptors (Fig 1b) (reviewed in Hochachka and Mustafa, 1972; Müller et al., 2012). This transfer of the electrons out of the ETC and onto alternative electron acceptors requires the quinone-coupled dehydrogenases (Kita, 1992; Ma et al., 1993). Under aerobic conditions these QDHs act as entry points to the ETC, transferring electrons from their substrates to UQ. Crucially, the reactions catalysed by these QDHs are reversed in anaerobic conditions - they now act as reductases transferring electrons out of the ETC and onto their products. For example, Complex II acts as a succinate dehydrogenase in aerobic conditions, generating fumarate; in anaerobic conditions, it reduces fumarate generating succinate as a terminal electron sink (Fig 1c) (Klockiewicz et al., 2002; Kmetec and Bueding, 1961; Sato et al., 1972; Saz and Vidrine, 1959; Takamiya et al., 2002). In this way an entry of electrons into the ETC from a variety of electron donors in aerobic conditions is reversed to provide an exit from the ETC onto a variety of electron acceptors in anaerobic conditions.

The unusual ETC wiring used by STHs to survive anaerobic conditions requires an unusual electron carrier, Rhodoquinone (RQ) (Moore and Folkers, 1965). RQ and UQ are highly related molecules - the sole difference is the presence of an amine group on the quinone ring of RQ (Fig 1d). This changes the biophysical properties of the quinone ring: while UQ can accept electrons from the QDHs as they flow into the ETC under aerobic conditions, UQ cannot carry electrons of the correct electropotential to drive the reverse reactions in anaerobic conditions (Fioravanti and Kim, 1988; Sato et al., 1972). RQ can carry such electrons (Fioravanti and Kim, 1988; Sato et al., 1972), however, and the ability of STHs to survive in their hosts is absolutely dependent on RQ. The single amine group that differs between UQ and RQ thus affects the health of over a billion humans. RQ is found in very few animal species - only nematodes, molluscs and annelids are known to make RQ (Allen, 1973; Fioravanti and Kim, 1988; Klockiewicz et al., 2002; Sato and Ozawa, 1969; Takamiya et al., 2002; Van Hellemond et al., 1995). Since no host animals make RQ, inhibiting RQ synthesis or RQ use is a potentially powerful way to target parasites inside their host. Currently however little is known about RQ synthesis. The most mature studies have focused on the purple Proteobacterium R.rubrum, where RQ appears to derives from UQ (Brajcich et al., 2010). RQ synthesis in R.rubrum requires the gene rquA (Lonjers et al., 2012) which is the first and thus far only gene known to be required for RQ synthesis in any organism. It is still unclear what role it plays in RQ synthesis (Lonjers et al., 2012), nor what the rest of the genes required for RQ synthesis may be. In animals, the situation is even more blank: nothing is known about which genes are required for RQ synthesis and there are no drugs that are known to prevent RQ synthesis. This is partly because no

98 tractable animal model has been established in which to study RQ synthesis and use.

99 Previous studies have shown that C.elegans, a free-living helminth, can make RQ (Takamiya et al., 2002) and that when C.elegans is exposed to hypoxic conditions it undergoes major 
101

102

103

104

105

106

107

108

109

110

metabolic changes that resemble those that occur in STHs when they are in the hypoxic environment of their hosts (Butler et al., 2012; Föll et al., 1999). This suggested to us that we could establish C.elegans as a model for dissecting the pathway of RQ synthesis and for screening for drugs that block RQ synthesis or use. We confirm that C.elegans makes RQ and also that it uses RQ-dependent metabolism when unable to use oxygen as a terminal electron acceptor. Crucially, we identify the pathway of RQ synthesis in C.elegans and show that C.elegans requires RQ to survive under conditions where oxygen cannot be used as an electron acceptor for the ETC. This allowed us to establish a robust high throughput screening assay to identify compounds that block RQ synthesis or RQ use. This is the first study to show how RQ, an electron carrier that affects the life of over a billion humans, is made in helminths. This will help towards the development of a new class of drugs to treat these major human pathogens.

\section{Results}

\section{C.elegans makes RQ and switches to anaerobic metabolism when exposed to Potassium}

\section{Cyanide}

C.elegans is a non-parasitic helminth that is easily genetically tractable (Brenner, 1974; reviewed in Jones et al., 2005) and can be used for efficient drug screens (Burns et al., 2015). We wanted to establish C.elegans as a model to dissect the pathway for RQ synthesis in helminths and as a system in which we could efficiently screen for drugs that block the synthesis of RQ or use of RQ. We note that there are no other standard model organisms where this is possible: yeasts, insects, fish, and vertebrates do not make or use RQ so C.elegans is the sole genetically tractable animal model for this work.

Like other helminths, C.elegans has previously been shown to make RQ (Takamiya et al., 2002).

We wanted to confirm this and determine whether we could define a simple experimental method to drive C.elegans to carry out similar anaerobic metabolism as that used by parasitic helminths to survive in their hosts. We extracted quinones as described in Methods and as shown in Fig 2a and Supp Fig 1, C.elegans makes both UQ and RQ when maintained in normoxic conditions. We can therefore use C.elegans to genetically dissect the pathway for RQ synthesis.

Our next step was to establish a simple method to drive C.elegans to use RQ-dependent metabolism that would allow high throughput drug screens. Parasitic helminths use RQdependent metabolism under low oxygen conditions (Rioux and Komuniecki, 1984; Saz and Lescure, 1969; Tielens et al., 1992) and previous studies showed that C.elegans shows similar metabolic shifts when exposed to hypoxic conditions (Butler et al., 2012; Föll et al., 1999). If possible, however, we wanted to avoid the use of hypoxic chambers. While hypoxia chambers are highly accurate ways of controlling oxygen levels, they are also very expensive and cumbersome for large-scale drug screens. We therefore turned to chemical methods of inducing a hypoxic state. Potassium Cyanide (KCN) is a potent inhibitor of Complex IV (Antonini et al., 
1971; Nicholls et al., 1972) - KCN inhibits oxygen binding to Complex IV and KCN treatment thus mimics hypoxia. We tested whether treatment with $\mathrm{KCN}$ could drive C.elegans to use anaerobic metabolism that is similar to the RQ-dependent metabolism used by STHs in their hosts. The classic hallmark of RQ-dependent anaerobic metabolism in helminths is the generation of high levels of succinate through the reversal of Complex II (Fig 1c) (Butler et al., 2012; Saz and Lescure, 1969; Tielens et al., 1992). If C.elegans can indeed use the same anaerobic metabolism as parasitic helminths, there should be a build-up of succinate following KCN treatment. Furthermore, this should be dependent on Complex I activity, since Complex I is the sole source of electrons that are carried by RQ to drive the fumarate reductase activity of Complex II (Fig 1b). We found that when C.elegans are exposed to KCN, they build up high levels of succinate as expected and that inhibiting Complex I with rotenone prevents succinate build-up (Fig 2b). We thus find that C.elegans makes RQ and that treatment of C.elegans with $\mathrm{KCN}$ causes them to switch to a metabolic state that resembles that of STHs in their host. C.elegans is thus an excellent model in which to dissect RQ synthesis and to screen for compounds that alter RQ-dependent metabolism.

\section{In C.elegans, RQ is synthesised from products of the kynurenine pathway and not from}

\section{Ubiquinone}

RQ and UQ are highly related molecules - the sole difference is the presence of an amine group on the quinone ring of RQ (Fig 1d). The critical question for RQ synthesis is where this amine group comes from and how it is generated. The best-defined current model for RQ synthesis comes from experiments in the proteobacterium R.rubrum. At least in this prokaryote, RQ is thought to be made by a late addition of the critical amine group to an existing molecule of UQ (Brajcich et al., 2010). UQ is thus an obligate precursor of RQ and RQ synthesis requires initial synthesis of UQ (Fig 3a). While this may be the case for R.rubrum, this is not the case in F.hepatica (Van Hellemond et al., 1996) or C.elegans. The clk-1(qm30) strain has a loss-offunction mutation in the C.elegans COQ7 orthologue that is required for hydroxylation of 5demethoxyubiquinone to 5-hydroxyubiquinone, a late step in UQ synthesis - there is no detectable UQ in clk-1 (qm30) homozygous animals. However, a previous study showed that there does appear to be RQ in this strain (Jonassen et al., 2001). If there is no UQ, but there is RQ, then RQ is not derived from UQ, at least in helminths. The two models for RQ synthesis thus differ fundamentally — in one UQ is an obligate precursor (Brajcich et al., 2010), in the other it is not (Jonassen et al., 2001). Since this is a fundamental result, we wanted to confirm this before trying to dissect the pathway of RQ synthesis. We thus extracted and analysed quinones from either wild-type worms or clk-1(qm30) homozygous animals. We find that while there is no detectable UQ in clk-1(qm30) mutants, there is abundant RQ and indeed we find that RQ levels are essentially unchanged (Fig 3b). We thus confirm that UQ is not an obligate

177 If RQ is not generated by addition of the key amine group to an existing UQ molecule where 178 does the amine group on RQ come from? One possibility is it is added not to UQ but to a UQ 
179 precursor such as demethoxyquinone (DMQ) - such UQ precursors would still be present in the

180 clk-1 (qm30) mutant strain (Fig 3a for schematic). While this could in principle be the case, it is

181 unlikely because amination of an aromatic ring is highly thermodynamically unfavorable

182 (reviewed in Downing et al., 1997). We therefore investigated an alternative possibility — that

183 the critical amine group of RQ is not added in a late step of RQ synthesis but instead is present

184 from the outset.

185 A key initial step in UQ synthesis is the addition by COQ-2 of a polyprenyl tail to a $p$ -

186 hydroxybenzoate ring (PHB - also often called 4-hydroxybenzoate (4-HB)) (Momose and

187 Rudney, 1972; Trumpower et al., 1974). PHB has no amine group - however, S.cerevisiae

188 COQ2 is known to be able to use a variety of similar compounds as substrates for prenylation

189 such as para-aminobenzoic acid and vanillic acid (reviewed in Pierrel, 2017). Given the potential

190 substrate flexibility of COQ-2, we hypothesized that RQ synthesis might start not with PHB but

191 with a related molecule that contains an amine group already on the ring (Fig 3 for schematic). In

192 particular, we noted that yeast COQ2 is tolerant of substituents at positions 5 and 6 of the PHB

193 structure (reviewed in Pierrel, 2017) suggesting that this might be feasible enzymatically. We

194 considered different candidate molecules as amine-containing ring structures that might act as

195 precursors for RQ and focussed on anthranilate and 3-hydroxyanthranilate (3HA) as likely

196 sources of the amine-containing ring in RQ. Anthranilate and 3HA are made from the amino acid

197 tryptophan via the kynurenine pathway (Heidelberger and Gullberg, 1948; Kotake, 1936)and

198 kynu-1 encodes the C.elegans kynureninase that is required for the generation of anthranilate and

199 3HA(Babu, 1974; Bhat and Babu, 1980; van der Goot et al., 2012). We examined the quinones

200 present in kynu-1(e1003) mutants that lack kynureninase and while UQ levels are normal in the

201 kynu-1(e1003) mutant animals, there is no detectable RQ (Fig 3c). This suggests that the amine

202 group on RQ derives from anthranilate or 3HA, or some closely related product of kynureninase.

203 To further confirm that the amine group on RQ ultimately derives from tryptophan we tested

204 whether a tryptophan-derived aromatic amino group is being incorporated into RQ. We fed

205 C.elegans $15 \mathrm{~N}$-labelled bacteria for 3 generations either in the presence or absence of $14 \mathrm{~N}$

206 tryptophan. As shown in Fig 4a, the sole source of any $14 \mathrm{~N}$ incorporated into RQ is the $14 \mathrm{~N}$

207 tryptophan. As expected, the RQ detected in animals fed with $15 \mathrm{~N}$ bacteria alone is

208 approximately all $15 \mathrm{~N}$ labelled (Fig 4b). However, if $14 \mathrm{~N}$ tryptophan was added, $250 \%$ of the

209 RQ observed was 14N RQ - the sole source for this $14 \mathrm{~N}$ was the added tryptophan. Taken

210 together, our data show that RQ does not derive from UQ, and that anthranilate, 3HA, or a

211 related molecule deriving from tryptophan via the kynurenine pathway is the source of the amine

212 group on the quinone ring of RQ. We propose that the pathway of RQ and UQ synthesis are

213 largely the same - the key difference is the presence or absence of the amine group on the

214 initial aromatic ring substrate for COQ-2.

215

216 RQ is required for long-term survival of C.elegans in anaerobic conditions 
As shown in Fig 2, C.elegans shows similar changes in metabolism when it is treated with KCN as STHs undergo when they adapt to the hypoxic environment of their host. For example, the classic hallmark of this RQ-dependent anaerobic metabolism is the generation of succinate by the action of Complex II as a fumarate reductase and C.elegans shows high levels of succinate when treated with KCN (Fig 3b). To confirm that this generation of succinate is indeed RQdependent in C.elegans, we examined whether RQ-deficient kynu-1(e1003) mutant animals could generate succinate when exposed to KCN. While wild-type worms generate high levels of succinate when Complex IV is inhibited with KCN, RQ-deficient kynu-1(e1003) mutant animals do not (Fig 5), confirming that the metabolic shift we see when we expose C.elegans to $\mathrm{KCN}$ is not simply similar to that of parasitic helminths in their hosts, it also requires RQ.

227 We have thus established that treating C.elegans with $\mathrm{KCN}$ drives them into an alternative metabolic state where they use RQ to drive the same anaerobic metabolism used by STHs in their hosts. To be able to screen efficiently for drugs that affect RQ synthesis or RQ-dependent metabolism, however, we need a direct phenotypic readout for RQ-utilization rather than a molecular readout (such as succinate generation). When do C.elegans require RQ-dependent metabolism and what are the consequences if they have no RQ? Since RQ-dependent metabolism is being used in the presence of $\mathrm{KCN}$ we compared the sensitivity of wild-type worms and kynu-1(e1003) mutants to KCN and the ability of wild-type worms and kynu1(e1003) mutants to survive in $\mathrm{KCN}$ for long periods. We found no significant differences in acute KCN sensitivity of wild-type worms and kynu-1(e1003) mutants - both slow their movement in the presence of $\mathrm{KCN}$, stop moving completely by $\sim 90$ minutes (Fig 6a), and remain immobile from there on when maintained in $\mathrm{KCN}$. However, there was a dramatic difference in their ability to survive extended periods in $\mathrm{KCN}$. We exposed worms to KCN for different lengths of time and then removed animals from KCN and assayed their movement over the next $3 \mathrm{hrs}$ as they recover from $\mathrm{KCN}$ treatment. When wild-type worms are removed from $\mathrm{KCN}$, they rapidly recover movement (Fig 6b) - they can do this even after 24 hours of KCN treatment (Fig 6c). However, kynu-1(e1003) mutants show greatly reduced ability to survive extended $\mathrm{KCN}$ treatment - they do not survive exposure to KCN for 12 hours or more (Fig 6c). RQdependent anaerobic metabolism thus allows C.elegans to survive extended periods where it cannot use oxygen as the terminal electron acceptor of the ETC.

This provides a simple assay for drugs that specifically affect RQ-dependent metabolism: drugs that block RQ synthesis or the activity of RQ-dependent pathways should abolish the ability of worms to survive $>12$ hours in KCN. To test this, we used the compound wact- 11 - this is a Complex II inhibitor that binds to the quinone-binding pocket of Complex II (Burns et al., 2015). wact-11 is highly related to the anthelmintic flutolanil (Burns et al., 2015) and is highly selective for helminth Complex II (Burns et al., 2015). Complex II is critical for RQ-dependent anaerobic metabolism where it acts as a fumarate reductase — inhibitors of Complex II might thus alter survival in KCN. That is what we observe: treatment with wact-11 prevents worms from surviving long-term $\mathrm{KCN}$ exposure (Figure 6d). Our assay will thus allow efficient screens for 
256 drugs that inhibit RQ synthesis or RQ utilization in vivo in a helminth under conditions where

257 they require RQ, the first time this has been possible.

258 Finally, we took advantage of a set of mutant worm strains that are resistant to wact-11

259 treatment. Mutations that result in resistance to wact-11 treatment cluster in the quinone binding 260 pocket of Complex II (Burns et al., 2015) and we reasoned that some of these might specifically 261 disrupt the binding of RQ and thus affect the ability of worms to survive extended exposure to $262 \mathrm{KCN}$. We tested a number of point mutants that affect wact-11 sensitivity (Fig 7a; data not 263 shown) and found that most mutants appear similar to wild-type worms in their ability to survive 264 long term KCN exposure. However, we found that the G71E mutation results in worms that are unable to survive extended KCN exposure - the G71E animals thus resemble kynu-1(e1003) mutants. We note that this mutation sits right above the modelled binding site for the rhodoquinone ring, whereas a neighbouring mutation that sits two turns of an alpha-helix further away (C78Y) has no effect. We thus suggest that the G71E mutation affects the ability of C.elegans to bind RQ into the quinone binding pocket of Complex II and thus to drive RQ dependent fumarate reduction as part of its RQ-dependent anaerobic metabolism.

\section{Discussion}

273 RQ was first identified over 50 years ago (Moore and Folkers, 1965). It is absolutely required for the survival of parasitic helminths in the hypoxic environment of the host gut where they can thrive for many months. The single amine group that differs between RQ and UQ is crucial for this - it allows RQ to carry electrons of the right electropotential to drive quinone-coupled dehydrogenases (QDHs) in reverse, acting as reductases (Fioravanti and Kim, 1988; Sato et al., 1972). In aerobic conditions, QDHs carry electrons from a diverse set of electron donors and transfer them onto UQ and hence into the ETC; under anaerobic conditions, RQ carries electrons to the QDHs which then reduce a diverse set of electron sinks, providing an exit point for electrons from the ETC. The single amine group on the quinone ring of RQ allows parasites to carry out this unusual anaerobic metabolism and thus it affects the lives of over a billion humans. Despite the importance of RQ for human health, its synthesis has been elusive and no anthelmintics have been identified that affect RQ synthesis. Here, we used C.elegans genetics to identify the RQ synthesis pathway and to establish a pipeline for screening for new compounds that alter the ability of worms to make and use RQ.

287 The critical question in RQ synthesis is where the critical amine group on the quinone ring 288 comes from and how it is added. Previous studies suggested that RQ is synthesised using UQ as 289 a precursor (Brajcich et al., 2010) and that the amine group is added at a late stage in RQ 290 synthesis. Here, we show that this is not true, at least in C.elegans. We show that RQ synthesis 291 does not require UQ as a precursor and, crucially, that the critical amine group on the quinone 292 ring of RQ is not added at a late stage in the synthesis of RQ as has been previously proposed 293 (Brajcich et al., 2010) but that it is present from the initial steps of RQ synthesis. For the first time since its discovery in the early 1960s, we now have a key insight into how RQ is made in 
helminths and this has several implications for the search for novel anthelmintics that might affect RQ synthesis.

297 First, we do not believe that there are separate dedicated pathways for UQ and for RQ synthesis 298 in helminths. Instead, we suggest that UQ and RQ have a largely shared synthesis pathway. The 299 key difference in RQ and UQ synthesis is the use of different initial substrates for COQ-2: if 300 PHB is used, the product will be UQ, if anthranilate, 3HA, or a related product of tryptophan metabolism is used, the product will be RQ. This stands in clear contrast to the pathways used by a different set of organisms to make two different quinones, one for aerobic and one for anaerobic metabolism: facultative anaerobic bacteria including non-pathogenic E.coli as well as major human pathogens like M.tuberculosis. These bacteria make two quinones: UQ which is used as an electron carrier under aerobic conditions, and menaquinone (MK) (reviewed in Kwon and Meganathan, 2009), which acts as a carrier under anaerobic conditions and is in some sense analogous to RQ as it can carry electrons that can drive fumarate reduction (Cecchini et al., 1986). The synthesis pathways of UQ and MK are completely distinct and the genes involved are distinct (reviewed in Kwon and Meganathan, 2009). This separation of MK and UQ synthesis pathways has allowed the development of a number of promising compounds that act as inhibitors of the MK synthesis pathway (reviewed in Boersch et al., 2018). We suggest that there may be no analogous inhibitors for 'the RQ synthesis pathway' in helminths since there does not appear to be a dedicated RQ pathway analogous to the dedicated MK synthesis pathway.

314 Second, the pathway we identify for RQ synthesis suggests novel targets for anthelmintics. The finding that the kynurenine pathway is the source of the key precursors for RQ synthesis suggests naively that helminth-specific inhibitors of the kynureninase pathway might act as potent anthelmintics. However, the human gut is likely to be a source of anthranilate and 3HA from host metabolism or from the microbiome and inhibiting production of these molecules in the helminth itself might thus prove ineffective. A more likely target is COQ-2, the enzyme that prenylates the anthranilate or 3HA ring as the first step in RQ synthesis. Again, it is worth drawing an analogy between the synthesis pathways of UQ and MK in bacteria. The enzymes responsible for the prenylation steps of UQ and MK synthesis are entirely distinct and act at very different steps - UQ prenylation by ubiA (the E.coli coq-2 orthologue) is a very early step, the MK prenylation by men $A$ is a late step in MK synthesis (reviewed in Kwon and Meganathan, 2009). Inhibitors of menA (reviewed in Debnath et al., 2012; Kurosu et al., 2007) have no impact on $u b i A$ or human COQ2 activity since menA and UbiA/COQ2 are completely different enzymes. Inhibitors that specifically block RQ synthesis in helminths while leaving host UQ synthesis intact will need to be more selective since they target orthologous enzymes, the human and helminth COQ2. However, since they have different substrate specificity, this may be possible and opens up a potential avenue for new anthelmintics. so rare amongst animals. To date, only three groups of animals are known to make RQ: molluscs, annelids, and helminths (Allen, 1973; Fioravanti and Kim, 1988; Klockiewicz et al., 2002; Sato and Ozawa, 1969; Takamiya et al., 2002; Van Hellemond et al., 1995). If RQ 
synthesis and UQ synthesis largely share a common pathway, why doesn't every animal that makes UQ also make RQ? One possibility is that while much of the pathway for UQ and RQ synthesis is shared, RQ synthesis requires additional components that might only be present in RQ-synthesising species. We find some evidence that this may be the case.

339 The sole gene known to be required specifically for RQ synthesis and not for UQ synthesis is the R.rubrum gene rquA (Lonjers et al., 2012). This is a methyltransferase that is related to the quinone methyltransferases UbiG/COQ3 and UbiE/COQ5 that act in UQ synthesis (Lonjers et al., 2012; Stairs et al., 2018). Although RquA is predicted by homology to act as a quinone methylase (Lonjers et al., 2012), its exact role in RQ synthesis is still obscure and no clear orthologues in helminths or other RQ-producing animals have been identified to date. Whatever the function of RquA, it is clear that R.rubrum has three distinct quinone methyltransferases two that are required for UQ synthesis and the third required specifically for RQ synthesis (Lonjers et al., 2012; Stairs et al., 2018). All animal genomes encode orthologues of UbiG/COQ3 and $\mathrm{UbiE} / \mathrm{COQ} 5$ - these are the sole genes predicted to encode quinone methyltransferases in almost all animal genomes and there is no third related set like in R.rubrum. Helminths, molluscs, and annelids, the animal species known to make RQ, are different however - in addition to COQ3 and COQ5, they encode an additional set of related quinone methyltransferases (Fig 8a). In C.elegans this comprises four paralogues, R08F11.4, R08E5.1, $R 08 E 5.3$ and K12D9.1, and we find orthologues of these additional quinone methyltransferases in all parasitic helminths examined so far (examples in Fig 8a). Three of these additional quinone methyltransferase paralogues (R08F11.4, R08E5.1 and R08E5.3) are highly upregulated in C.elegans mutants that are homozygous for a loss of function mutation in gas- 1 which encodes a subunit of Complex I in the ETC - R08E5.3 is in the top 50 most upregulated genes, and all three are in the top $4 \%$ of upregulated genes (Falk et al., 2008). This suggests that their function may be required when aerobic respiration is compromised, as would be expected if they play a role in RQ synthesis. This correlation between expression of these additional quinone methyltransferases and RQ-dependent metabolism also appears to hold in parasites. For example, in Ascaris, RQ-dependent metabolism is essential for the survival of the adult worm and this is largely occurring in the muscle cells (Saz and Lescure, 1969). We identified an Ascaris orthologue of this additional class of quinone methyltransferases, $\operatorname{AgB0} \_\mathrm{g} 209$, that shows low expression in most developmental stages, but is strongly upregulated specifically in adult muscle cells (Fig 8b) (Jex et al., 2011). Thus while most animals only encode COQ3 and COQ5, RQsynthesizing animal species encode an additional set of quinone methyltransferases whose expression is strongly upregulated under conditions requiring anaerobic respiration. This suggests that there may be similarity in RQ synthesis in R.rubrum and animals and that there may be at least some genes with functions specific to RQ synthesis. It will be interesting to examine whether these additional quinone methyltransferases participate in RQ synthesis in

373 sequence similarity. 
374 Finally, we note that several steps in the kynurenine pathway and in the ubiquinone synthesis

375 pathway are catalysed by either monooxygenases or dioxygenases that require oxygen. These

376 include TDO-2 (Hayaishi et al., 1957) and KMO-1 (Detmer and Massey, 1985; Entsch et al.,

377 1976) in the kynurenine pathway and COQ-6 (Ozeir et al., 2015) and CLK-1 (Marbois and

378 Clarke, 1996) in the UQ synthesis pathway. RQ synthesis thus appears to require the availability

379 of oxygen for these enzymes, an unexpected result since RQ is preferentially required in

380 anaerobic conditions and is the predominant quinone in helminths living under anaerobic

381 conditions. How might these oxygen-requiring steps be carried out for RQ synthesis? It is

382 possible that the helminth enzymes have evolved so that they can still operate under low oxygen

383 conditions. Other oxygen-using proteins have evolved extremely high oxygen affinity in

384 helminths - for example Ascaris haem is octameric and binds oxygen with 25,000 times

385 greater affinity than human haem (Minning et al., 1999). Alternatively, these same enzymatic

386 steps might be carried out by other enzymes in lower oxygen conditions. In E.coli, for example,

387 UbiB and UbiF (reviewed in Kwon and Meganathan, 2009) carry out the same hydroxylation

388 modifications to the quinone ring as COQ-6 and CLK-1 in aerobic conditions and mutation of

389 either gene results in a lack of mature UQ in these bacteria. However, under anaerobic

390 conditions, $u b i B$ and $u b i F$ mutants make normal levels of UQ suggesting that other enzymes

391 carry out these reactions in low oxygen conditions (Alexander and Young, 1978). It is possible

392 that there is an analogous set of enzymes that are required for RQ synthesis in low oxygen

393 conditions - these would carry out similar reactions to COQ-6 and CLK-1 but without the

394 requirement for oxygen.

395 There is thus still much to be discovered about the regulation and the precise pathway of RQ

396 synthesis in helminths. The results presented here provide a firm starting point and the assay we

397 describe for drugs that affect RQ-dependent metabolism may lead to the discovery and

398 development of a new class of anthelmintic drugs. Since resistance to known classes of

399 anthelmintics is widespread among livestock parasites like H.contortus, C.oncophora and

400 A.suum and is rising in human populations (reviewed in Sangster et al., 2018), this will prove

401 critical in the control and treatment of these major pathogens. 


\section{Materials and Methods}

\section{Worm Strains and Maintenance}

405 In addition to the traditional laboratory strain N2, here we include work using strains $c l k$ -

406 1(qm30), kynu-1(e1003), sdhc-1(tr357), and sdhc-1(tr423). The two sdhc-1 strains were provided

407 by Dr. Peter Roy and all other strains were provided by the Caenorhabditis Genetics Centre. All

408 worms were maintained on NGM agar plates seeded with E. coli OP50 as described elsewhere

409 (Stiernagle, 2006) and maintained at $20^{\circ} \mathrm{C}$.

\section{RQ Tryptophan Anti-Labelling Experiment}

411 Escherichia coli (MG1655) was grown overnight at $37^{\circ} \mathrm{C}$ in $\mathrm{M} 9$ media prepared using $1 \mathrm{~g} / \mathrm{L}{ }^{15} \mathrm{~N}$

412 ammonium chloride (Cambridge Isotopes) as the nitrogen source. Bacteria were heat killed at

$41365^{\circ} \mathrm{C}$ for 15 minutes. The heated culture was used to seed NGM agar plates. $500 \mu \mathrm{L}$ of $50 \mathrm{mg} / \mathrm{ml}$

414 tryptophan in water was spread on each $10 \mathrm{~cm}$ plate. Ten L4 nematodes were placed on each

415 plate to lay eggs overnight at $20^{\circ} \mathrm{C}$. Adult worms were removed following the egg laying period.

416 After 5 days at $20^{\circ} \mathrm{C}$ the nematodes were collected and frozen at $-80^{\circ} \mathrm{C}$.

\section{Quinone Extraction}

418 Nematode samples were thawed and lysed via sonication. Quinone extraction solvent containing 419 a 2:1 ratio of chloroform and methanol (Thermo Fisher Optima LC-MS grade) respectively was 420 added to the samples. The organic phase of the sample was collected and then dried using 421 nitrogen gas. Samples were resuspended in a 60:40 acetonitrile and isopropanol solution prior to 422 analysis using APCI LC-MS.

\section{Quinone LC-MS analysis}

424 Quinones were analyzed by reverse phase chromatography on an Eclipse Plus C-18 RRHD 425 column, $2.1 \mathrm{mM}$ x $50 \mathrm{~mm}$ with 1.8 um packing operated in a thermostatted column compartment 426 held at $70^{\circ} \mathrm{C}$. Buffer A was $50 \% \mathrm{MeCN}$ in water, Buffer B was $100 \%$ acetone with $0.01 \%$

427 formic acid. Starting conditions were $0.25 \mathrm{~mL} / \mathrm{min}$ at $50 \%$ B. Gradient was 1-minute hold, 428 followed by increase $100 \%$ B at 5 minutes, hold $100 \%$ B until 7 minutes, then return to $50 \%$ B at 4297.1 minutes and hold until 10 minutes. Samples were introduced from a HTC pal by injection of $4305 \mu \mathrm{L}$ sample into a $2 \mu \mathrm{L}$ loop. Wash 1 was acetonitrile and wash 2 was isopropanol. Samples 431 were ionized using a Multimode ionization source (Agilent) operated in APCI mode, gas temp $432350^{\circ} \mathrm{C}$, vaporizer temp $350^{\circ} \mathrm{C}$, drying gas $5 \mathrm{~L} / \mathrm{min}$, nebulizer $60 \mathrm{PSI}$, capillary voltage $4000 \mathrm{~V}$, 433 corona current $4 \mu \mathrm{A}$, skimmer voltage $70 \mathrm{~V}$, octupole $1 \mathrm{RF} 400 \mathrm{~V}$. Samples were analyzed on a 4346230 TOF, a 6545 Q-TOF, or a 6490 QQQ as indicated. Fragmentor voltage for TOF/QTOF 435 analysis was $200 \mathrm{~V}$. For QQQ analysis, ubiquinone 9 was monitored by MRM of 795.6/197.3 at 436 CID of $52 \mathrm{~V}$; rhodoquinone-9 was monitored at 780.6/192.1 at CID of $52 \mathrm{~V}$.

\section{Image-based assays}

438 All image-based experiments were conducted on L1 animals which were collected from mixed439 stage plates and isolated using a 96 well $11 \mu \mathrm{M}$ Multiscreen Nylon Mesh filter plate (Millipore: 
S5EJ008M04) as described previously (Spensley et al., 2018) to a final concentration of $\sim 100$ animals per well. They were then incubated in a final concentration of $200 \mu \mathrm{M}$ potassium cyanide for varying amounts of time. There are two key assays: the acute and the recovery. The acute assay monitors worm movement immediately following exposure to KCN every 5 minutes for a total of 3 hours. The recovery assays involve a KCN incubation of 3, 6, 9, 12, or 18 hours after which the $\mathrm{KCN}$ is diluted 6-fold with M9 buffer. Immediately after dilution, worm movement is monitored every 10 minutes for 3 hours. In both assays, worm movement is quantified using an image-based system as previously described (Spensley et al., 2018). All data were normalized by the fractional mobility score of the M9-only control wells per strain per time point.

\section{Drug preparation and Assay assembly}

451 Solutions of potassium cyanide (Sigma 60178-25G) were made fresh prior to each experiment in phosphate buffered saline (PBS) and then diluted to a $5 \mathrm{mM}$ stock solution in M9 buffer. $2 \mathrm{X}$ working concentrations were then prepared with $\mathrm{M} 9$ and the $\mathrm{KCN}$ stock solution. Wact-11 (Chembridge ID 6222549) was kept frozen as a $100 \mathrm{mM}$ stock in DMSO. Diluted wact-11 stocks were made in DMSO (BioShop DMS666) to a concentration of $3.75 \mathrm{mM}$ and kept frozen until day of use. 10X working concentrations were made with wact-11 stock, M9 buffer, and DMSO. All experiments were prepared to contain $0.8 \% \mathrm{v} / \mathrm{v}$ DMSO to control for any confounding effects of drug solvent.

Assays were assembled in flat-bottomed polystyrene 96-well plates (Corning 3997) to a total volume of $100 \mu \mathrm{L}$ and $40 \mu \mathrm{L}$ for the acute and recovery assays, respectively. Apart from assays including wact-11 which constituted half $\mathrm{KCN}$ solution, $10 \%$ wact- 11 solution, and $40 \%$ worms in buffer, all other assays were comprised of equal parts worms in buffer and KCN solution.

\section{Rotenone LC-MS}

Worms were collected and isolated as described above. A final concentration of $7.5 \mathrm{~L} 1 \mathrm{~s} / 10 \mu \mathrm{L}$ was treated with final concentrations of $12.5 \mu \mathrm{L}$ rotenone (Sigma R8875) in $0.8 \% \mathrm{DMSO}$ and with $0.8 \%$ DMSO alone and with $100 \mu \mathrm{M} \mathrm{KCN}$ in M9 or with M9 alone, $20 \mathrm{~mL}$ altogether in 40 $\mathrm{mL}$ plastic containers (Blender Bottle 600271) and were on a shaker for $1 \mathrm{hr}$ at room temperature. After $1 \mathrm{hr}$, samples were poured over $0.2 \mu \mathrm{M}$ Nylaflo nylon filter membranes (PALL 66604) over vacuum and once the supernatant had run through, the filter paper was placed in prepared $1.2 \mathrm{~mL}$ of 8:1:1 extraction solvent (MeOH, HPLC Grade (SA 34860); $\mathrm{H}_{2} \mathrm{O}$, HPLC Grade (Caledon 8801-7-40); $\mathrm{CHCl}_{3}$, HPLC Grade (SA 650498)) in a $1.5 \mathrm{~mL}$ microfuge tube on dry ice. Tubes were inverted 5 times then vortexed. the tubes spun at $13,200 \mathrm{rpm}$ at $4^{\circ} \mathrm{C}$ for $30 \mathrm{~min} .1 \mathrm{~mL}$ of supernatant was transferred to a new tube and dried under dry $\mathrm{N}_{2}$ with $<0.02 \% \mathrm{O}_{2}$ at 5 PSI for $8 \mathrm{hrs}$. Each sample was reconstituted with $30 \mu \mathrm{L}$ HPLC grade water as was prepared labelled yeast reference. Samples and reference were spun at $13,200 \mathrm{rpm}$ at $4^{\circ} \mathrm{C}$ for $5 \mathrm{~min} .10 \mu \mathrm{L}$ sample and reference were placed in an LC-MS 
478 sample vial (Agilent 5190-2243, cap is Agilent 5185-5820) and were fast spun at 1,000 rpm at

$4794^{\circ} \mathrm{C}$.

480 kynu-1 LC-MS

$481 \quad \mathrm{~N} 2$ and kynu-1(e1003) worms were washed and filtered as previously described. They were then

482 placed in $1.5 \mathrm{~mL}$ microfuge tubes at $1.5 \mathrm{~mL}$ for a final concentration of $45 \mathrm{~L} 1 \mathrm{~s}, 300 \mu \mathrm{M} \mathrm{KCN}$ in

483 M9 or M9 alone and were placed on a rotator for 6 hours at room temperature. After 6 hours, the

484 samples were extracted and prepared as previously described.

\section{Succinate Analysis}

486 Succinate was extracted from an IPRP method LC-MS run at an mzCenter of 117.0193 and a 487 retention time of $690 \mathrm{~s}$ in the case of the kynu-1 experiment and from an Acid method LC-MS 488 run at an mzCenter of 117.0193 and a retention time of $141 \mathrm{~s}$ in the case of the rotenone 489 experiment. Both sets of samples were normalized to a labelled yeast reference. In the case of the 490 kynu-1 experiment, they were further normalized to the median of all the extracted peaks for 491 each sample. In both cases they were ultimately normalized to the mean unlabelled N2 sample 492 treated with buffer or $0.8 \%$ DMSO. Plots were generated using \{plotPeak\}.

\section{Structural Analysis}

494 Sequences of succinate dehydrogenase, subunit C from R. rubrum (UniProtKB: Q2RV42), E.coli 495 (UniProtKB: P0A8Q0 and P69054), C. gigas (UniProtKB: K1R921), C. elegans (Wormbase:

496 CE00598 and CE50785), A. suum (UniProtKB: F1LC27), S. cerevisiae (UniProtKB: P33421 and 497 Q04487), H. sapiens (UniProtKB: O14521), M. musculus (UniProtKB: Q9CZB0), S. scrofa 498 (UniProtKB: D0VWV4), M. balamuthi (UniProtKB: A0A0B5D2L2), A. ceylanicum 499 (UniProtKB: A0A0D6M6A9), W. bancrofti (UniProtKB: J9F801), L. loa (UniProtKB: 500 A0A1I7VAG2), A. simplex (UniProtKB: A0A0M3K8C7), O. bimaculoides (UniProtKB: A0A0L8IER1), A. vulgaris (UniProtKB: A0A0B7BS14), C. clemensi (UniProtKB: C1C048), $M$. 502 yessoensis (UniProtKB: A0A210PU64) underwent ClustalOmega multiple alignment (Sievers et 503 al., 2011) using the default settings on EMBL-EBI.

504 Mitochondrial rhodoquinol-fumarate reductase from A. suum bound with rhodoquinone-2 (PDB: 505 3VR8) was displayed on Chimera (Pettersen et al., 2004) and the C. elegans sequence was 506 threaded by homology using Modeller (Sali and Blundell, 1993; Webb and Sali, 2016) and the 507 MSA with 15 iterations.

508 Alignment of Quinone Methyltransferases

509 Sequences of COQ3/UbiG, Coq5/UbiE and the RQS cluster were identified by BLAST (Altschul 510 et al., 1990) in S. cerevisiae, H. sapiens, A. californica, M. yessoensis, C. telata, C. elegans, A. 511 ceylanicum and $N$. americanus. The sequences were then aligned by ClustalOmega (Sievers et 512 al., 2011) and the phylogenetic relationships are taken from ClustalOmega (Sievers et al., 2011). 
bioRxiv preprint doi: https://doi.org/10.1101/627737; this version posted May 4, 2019. The copyright holder for this preprint (which was not aCC-BY-NC 4.0 International license.

515 The research in this study was supported by CIHR grant 501584. We thank Prof. Peter Roy 516 and multiple members of Fraser and Roy labs for insightful discussions, Prof. Brent Derry for 517 intellectual stimulation, the Caenorhabditis Genetics Center for C.elegans strains, Olga Zaslaver 518 and Angela Wong for their timeand help wuth mass spec analyses. Structural graphics and 519 analyses performed with UCSF Chimera, developed by the Resource for Biocomputing, 520 Visualization, and Informatics at the University of California, San Francisco, with support from $521 \quad$ NIH P41-GM103311

522 
524 Figure 1: Anaerobic metabolism in helminths requires Rhodoquinone (RQ). a. Electron

525 flow in the Electron Transport Chain (ETC) under aerobic conditions. Electrons enter the

526 ETC either via Complex I or via a number of Quinone-coupled Dehydrogenases (QDH; cyan).

527 These complexes transfer electrons to Ubiquinone (red circle 'UQ') which shuttles them to

528 Complex III. They exit the ETC at Complex IV where they are transferred to oxygen as the

529 terminal electron acceptor. Proton pumping is coupled to electron transport and is carried out by

530 Complexes I, III and IV. Electron flow is shown in red and proton pumping in green. b. Electron

531 flow in the Electron Transport Chain (ETC) under anaerobic conditions. Electrons still

532 enter the ETC at Complex I which transfers electrons to RQ (cyan circle 'RQ'). RQ shuttles

533 electrons to the QDHs which now operate as reductases, allowing electrons to exit the ETC and

534 onto a diverse set of terminal electron acceptors. Complex I is the sole proton pump in this

535 truncated ETC. c. Schematic of Complex II activity under aerobic and anaerobic conditions.

536 Under aerobic conditions, Complex II acts as a succinate dehydrogenase, transferring electrons

537 from succinate onto UQ. Under anaerobic conditions, Complex II operates in the reverse

538 direction acting as a fumarate reductase, accepting electrons from RQ and transferring them to

539 succinate as the terminal electron sink. d. Structure of UQ and RQ. The critical amine group

540 differing between UQ and RQ is highlighted; the prenyl tail is shown schematically as a green

541 wavy line.

542 Figure 2: C.elegans makes Rhodoquinone (RQ) and can carry out RQ-dependent anaerobic

543 metabolism. a. C.elegans makes both UQ and RQ. C.elegans were grown under normoxic

544 conditions and quinones extracted and analysed by mass spectrometry (see Methods). Both UQ

545 and RQ can be detected. b. C.elegans increases succinate production following treatment

546 with Potassium Cyanide (KCN). C.elegans L1 larvae were treated either with $200 \mu \mathrm{M} \mathrm{KCN}$

547 alone, $12.5 \mu \mathrm{M}$ rotenone alone, or a combination of $\mathrm{KCN}$ and rotenone for $1 \mathrm{hr}$ and metabolites

548 extracted and analysed by mass spectrometry (see Methods). The graph shows that succinate

549 levels increase over 5-fold following $\mathrm{KCN}$ treatment and that this increase is blocked by

550 rotenone indicating that it requires Complex I activity. Data are from 3 independent biological

551 repeats; box plots show median and the interquartile range as well as individual datapoints.

552 Figure 3: RQ in C.elegans does not derive from UQ but from Tryptophan metabolites. a.

553 Schematic showing possible routes for RQ synthesis. Current models for RQ synthesis are

554 shown schematically in the top pathway: PHB is prenylated by COQ-2 at the start of the UQ

555 synthesis pathway and RQ either derives from UQ or from a UQ precursor - the amine group is

556 thus added at a late step. The lower pathway shows our proposed pathway. Rather than use PHB

557 as the substrate for COQ-2, RQ synthesis starts with prenylation of anthranilate or 3-

558 hydroxyanthranilate by COQ-2 (the 3 hydroxy group is denoted by a red asterisk). The amine

559 group is thus present from the start of RQ synthesis rather than being added at a late step and RQ

560 synthesis proceeds via the same pathway as UQ synthesis. b. UQ is not a required

561 intermediate for RQ synthesis. $c l k$-1 (qm30) mutant worms and N2 wild-type worms were

562 grown under normoxic conditions and quinones extracted and analysed. N2 worms contain both

563 UQ and RQ whereas $c l k-1$ (qm30) mutants only contain detectable RQ. c. RQ synthesis requires 
metabolism of Tryptophan via the kynurenine pathway. kynu-1(e1003) mutant worms were grown under normoxic conditions and quinones extracted and analysed. While wild-type worms contain both UQ and RQ, kynu-1(e1003) mutants only contain detectable UQ. RQ synthesis thus requires the generation of $3 \mathrm{HA}$ or anthranilate from Tryptophan via the kynurenine pathway.

Figure 4: The critical amine group on RQ derives from Tryptophan. a. Schematic of kynurenine pathway. Anthranilate and 3-hydroxyanthranilate (3HA) derive from Tryptophan via the kynurenine pathway and this requires KYNU-1 activity. The nitrogen atom that becomes part of the key amine group on RQ is highlighted. b. Analysis of RQ in animals fed either only $15 \mathrm{~N}$ substrates or $15 \mathrm{~N}$ substrates along with $14 \mathrm{~N}$ Tryptophan. Wild-type worms were fed with $15 \mathrm{~N}$-labelled bacteria for 3 generations either in the absence or in the presence of $14 \mathrm{~N}$ Tryptophan. Almost all RQ is $15 \mathrm{~N}$ labelled when worms were only eating $15 \mathrm{~N}$ bacteria. However when $14 \mathrm{~N}$ Tryptophan was also present, almost half the RQ is $14 \mathrm{~N} R Q$, indicating that the amine group of RQ must derive from Tryptophan. (N2) or kynu-1(e1003) mutant animals were exposed to $200 \mu \mathrm{M} \mathrm{KCN}$ for $6 \mathrm{hrs}$ and metabolites extracted and analysed by mass spectrometry. Data are from 5 independent biological repeats; box plots show median and the interquartile range as well as individual datapoints. Succinate levels increase markedly in $\mathrm{N} 2$ worms (red ' $\mathrm{N} 2$ ') following $\mathrm{KCN}$ treatment; there is no significant increase in $k y n u-1$ (e1003) mutant animals (cyan ' $k$ - 1 ') confirming that fumarate

583 reductase activity of Complex II requires RQ.

584 Figure 6: RQ-dependent metabolism is required for long-term survival in anaerobic conditions. a. Loss of RQ does not affect acute sensitivity to KCN. Wild-type (N2, red curve) and kynu-1(e1003) mutant L1 animals (kynu-1, blue curve) were exposed to $200 \mu \mathrm{M} \mathrm{KCN}$ and their movement measured over 3 hrs (see Methods). Both strains slow their movement and become immobile after $\sim 90$ mins. Curves show means of 3 biological replicates with 3 technical replicates in each; error bars are standard error. b. $\mathbf{R Q}$ is required for survival following extended treatment with KCN. Wild-type (N2, red curve) and kynu-1(e1003) mutant L1 animals (kynu-1, blue curve) were exposed to $200 \mu \mathrm{M} \mathrm{KCN}$ for $15 \mathrm{hrs}$. KCN was then diluted (see Methods) and worm movement was measured over a $3 \mathrm{hr}$ time course. Curves show means of 3 biological replicates with 3 technical replicates in each; error bars are standard error. c. Effect of different lengths of exposure to KCN on worm survival. Wild-type (N2, red) and kynu-1(e1003) mutant L1 animals (kynu-1, blue) were exposed to $200 \mu \mathrm{M} \mathrm{KCN}$ for different lengths of time from $3 \mathrm{hr}$ to $18 \mathrm{hr}$. KCN was then diluted (see Methods) and worm movement was measured after $3 \mathrm{hrs}$. Box plots show levels of movement after a $3 \mathrm{hr}$ recovery period. Data are from 3 biological replicates with 3 technical replicates in each. d. Ability to survive extended KCN exposure requires Complex II activity. Wild-type L1 animals were treated with $200 \mu \mathrm{M}$ alone, $10 \mu \mathrm{M}$ of wact-11 (a helminth-specific Complex II inhibitor) alone, or a combination of KCN and wact-11 for 15 hrs. Drugs were then diluted 6x and worm movement was measured over a $3 \mathrm{hr}$ timecourse. wact-11 treatment alone had no effect on survival over the experiment (black curve) and worms recovered completely from $\mathrm{KCN}$ treatment alone. However 
604 worms could not survive treatment with both KCN and wact-11. Curves show means of 3

605 biological replicates with 3 technical replicates in each; error bars are standard error.

606 Figure 7: Mutations in quinone-binding pocket of Complex II affect ability to survive 607 extended KCN treatment. a. Positions of mutations that alter wact-11 sensitivity. Structures

608 show either the wild-type residues or mutations that alter wact-11 binding. The structures shown 609 are C.elegans sequences (green) threaded onto the Ascaris suum crystal (pink). RQ is shown in 610 yellow with the critical amine group in blue - note that this is RQ2 and not RQ9, hence the 611 shortened prenyl tail. Note that G71E alters a residue that lies right over the quinone ring of RQ 612 whereas $\mathrm{C} 78 \mathrm{Y}$ is further from the ring. b. Effect of quinone-pocket mutations on ability to 613 survive extended KCN exposure. L1 worms containing either wild-type, G71E or C78Y mutant 614 mev-1 sequences were exposed to $200 \mu \mathrm{M} \mathrm{KCN}$ for $15 \mathrm{hrs}$. KCN was then diluted 6x and the 615 movement of worms measured across a $3 \mathrm{hr}$ time course. Curves show means of 3 biological 616 replicates with 3 technical replicates in each; error bars are standard error.

617 Figure 8: RQ synthesising species contain an additional class of quinone

618 methyltransferases. a. Eukaryotic quinone methyltransferases form three distinct classes.

619 We used BLAST to identify orthologues of UbiG/COQ3, UbiE/COQ5, and a novel additional 620 class of quinone methyltransferases in S. cerevisiae (Scer), H. sapiens (Hsap), the molluscs $A$. 621 californica (Acal) and M. yessoensis (Myes), the annelid C. telata (Ctel) and the helminths $C$. 622 elegans, A. ceylanicum, N. americanus (Cel, Acey, Name). Sequences were clustered using 623 ClustalOmega (Sievers et al., 2011) and form three distinct clades: UbiG/COQ3, UbiE/COQ5, 624 and the novel clade which we call RQS for RhodoQuinone Synthesis. b. Developmental

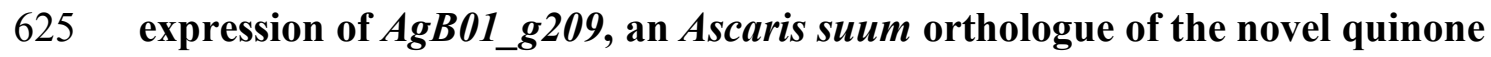
626 methyltransferases. (Jex et al., 2011) Expression levels (in TPM) are shown for L3 stages 627 (grown from isolated eggs in vitro (egg) or isolated from pig lung or liver), L4 stage larvae 628 isolated from gut, and reproductive tissue or muscle tissue isolated from either male or female 629 adult worms. Two adults are shown for each sample.

630 Supp Figure 1: Mass spectra for Rhodoquinone (RQ) and Ubiquinone (UQ). a. Mass 631 spectrometry (MS) extracted-ion chromatogram (EIC) of Rhodoquinone (RQ) (RT = $360 \mathrm{sec}$ ) 632 shown by a solid black line. An MS/MS EIC of the RQ product ion is shown with a dashed blue 633 line. b. MS EIC of Ubiquinone (UQ) (RT = $365 \mathrm{sec}$ ) shown by a solid black line. The dashed red 634 line represents the MS/MS EIC of Q's product ion. c. Mass spectra of RQ $(\mathrm{m} / \mathrm{z}=780.6)$ shown 635 in blue. Mass spectra of UQ ( $\mathrm{m} / \mathrm{z}=795.6)$ shown in red. d. Mass spectra of the RQ product ion $636(\mathrm{~m} / \mathrm{z}=182.1)$ shown in blue. Mass spectra of the $\mathrm{Q}$ product ion $(\mathrm{m} / \mathrm{z}=197.1)$ shown in red. 


\section{Bibliography}

639 Alexander K, Young IG. 1978. Alternative hydroxylases for the aerobic and anaerobic biosynthesis of ubiquinone in Escherichia coli. Biochemistry 17:4750-4755.

Allen PC. 1973. Helminths: Comparison of their rhodoquinone. Exp Parasitol 34:211-219. doi:10.1016/0014-4894(73)90080-5

Altschul SF, Gish W, Miller W, Myers EW, Lipman DJ. 1990. Basic local alignment search tool. $J$ Mol Biol 215:403-410. doi:10.1016/S0022-2836(05)80360-2

Antonini E, Brunori M, Rotilio GC, Greenwood C, Malmström BG. 1971. The Interaction of Cyanide with Cytochrome Oxidase. Eur J Biochem 23:396-400. doi:10.1111/j.14321033.1971.tb01633.x

Babu P. 1974. Biochemical genetics of Caenorhabditis elegans. Mgg Mol Gen Genet 135:39-44. doi:10.1007/BF00433899

Bhat SG, Babu P. 1980. Mutagen sensitivity of kynureninase mutants of the nematode Caenorhabditis elegans. MGG Mol Gen Genet 180:635-638. doi:10.1007/BF00268072

Boersch M, Rudrawar S, Grant G, Zunk M. 2018. Menaquinone biosynthesis inhibition: a review of advancements toward a new antibiotic mechanism. RSC Adv 8:5099-5105. doi:10.1039/C7RA12950E

Brajcich BC, Iarocci AL, Johnstone LAG, Morgan RK, Lonjers ZT, Hotchko MJ, Muhs JD, Kieffer A, Reynolds BJ, Mandel SM, Marbois BN, Clarke CF, Shepherd JN. 2010. Evidence that ubiquinone is a required intermediate for rhodoquinone biosynthesis in Rhodospirillum rubrum. J Bacteriol 192:436-445. doi:10.1128/JB.01040-09

Brenner S. 1974. The genetics of Caenorhabditis elegans. Genetics 77:71-94.

Brooker S, Clements ACA, Bundy DAP. 2006. Global Epidemiology, Ecology and Control of Soil-Transmitted Helminth Infections.Advances in Parasitology. Elsevier. pp. 221-261. doi:10.1016/S0065-308X(05)62007-6

Burns AR, Luciani GM, Musso G, Bagg R, Yeo M, Zhang Y, Rajendran L, Glavin J, Hunter R, Redman E, Stasiuk S, Schertzberg M, Angus McQuibban G, Caffrey CR, Cutler SR, Tyers M, Giaever G, Nislow C, Fraser AG, MacRae CA, Gilleard J, Roy PJ. 2015. Caenorhabditis elegans is a useful model for anthelmintic discovery. Nat Commun 6:7485-7485. doi: $10.1038 /$ ncomms 8485

Butler JA, Mishur RJ, Bokov AF, Hakala KW, Weintraub ST, Rea SL. 2012. Profiling the Anaerobic Response of C. elegans Using GC-MS. PLoS ONE 7:1-9. doi:10.1371/journal.pone.0046140

Cecchini G, Thompson CR, Ackrell BA, Westenberg DJ, Dean N, Gunsalus RP. 1986. Oxidation of reduced menaquinone by the fumarate reductase complex in Escherichia coli requires the hydrophobic FrdD peptide. Proc Natl Acad Sci U S A 83:8898-8902.

Crane FL, Hatefi Y, Lester RL, Widmer C. 1957. Isolation of a quinone from beef heart mitochondria. Biochim Biophys Acta 25:220-221.

Debnath J, Siricilla S, Wan B, Crick DC, Lenaerts AJ, Franzblau SG, Kurosu M. 2012. Discovery of selective menaquinone biosynthesis inhibitors against Mycobacterium tuberculosis. J Med Chem 55:3739-3755. doi:10.1021/jm201608g

Detmer K, Massey V. 1985. Effect of substrate and $\mathrm{pH}$ on the oxidative half-reaction of phenol hydroxylase. J Biol Chem 260:5998-6005.

Downing RS, Kunkeler PJ, van Bekkum H. 1997. Catalytic syntheses of aromatic amines. Catal Today 37:121-136. doi:10.1016/S0920-5861(97)00005-9

Entsch B, Ballou DP, Massey V. 1976. Flavin-oxygen derivatives involved in hydroxylation by 
684

685

686

687

688

689

690

691

692

693

694

695

696

697

698

699

700

701

702

703

704

705

706

707

708

709

710

711

712

713

714

715

716

717

718

719

720

721

722

723

724

725

726

727

728

729 p-hydroxybenzoate hydroxylase. J Biol Chem 251:2550-2563.

Falk MJ, Zhang Z, Rosenjack JR, Nissim I, Daikhin E, Nissim I, Sedensky MM, Yudkoff M, Morgan PG. 2008. Metabolic pathway profiling of mitochondrial respiratory chain mutants in C. elegans. Mol Genet Metab 93:388-397. doi:10.1016/j.ymgme.2007.11.007

Fioravanti CF, Kim Y. 1988. Rhodoquinone requirement of the Hymenolepis diminuta mitochondrial electron transport system. Mol Biochem Parasitol 28:129-134. doi:10.1016/0166-6851(88)90060-6

Föll RL, Pleyers A, Lewandovski GJ, Wermter C, Hegemann V, Paul RJ, Fo RL, Paul J. 1999. Anaerobiosis in the nematode Caenorhabditis elegans. Comp Biochem Physiol B Biochem Mol Biol 124:269-80.

Hayaishi O, Rothberg S, Mehler AH, Saito Y. 1957. Studies on oxygenases; enzymatic formation of kynurenine from tryptophan. J Biol Chem 229:889-896.

Heidelberger C, Gullberg ME. 1948. Concerning the mechanism of the mammalian conversion of tryptophan into kynurenine, kynurenic acid, and nicotinic acid. J Biol Chem 175:471.

Hochachka PW, Mustafa T. 1972. Invertebrate facultative anaerobiosis. Science 178:1056-1060.

Isom GE, Liu DH, Way JL. 1975. Effect of sublethal doses of cyanide on glucose catabolism. Biochem Pharmacol 24:871-875.

Jex AR, Liu S, Li B, Young ND, Hall RS, Li Y, Yang L, Zeng N, Xu X, Xiong Z, Chen F, Wu X, Zhang G, Fang X, Kang Y, Anderson GA, Harris TW, Campbell BE, Vlaminck J, Wang T, Cantacessi C, Schwarz EM, Ranganathan S, Geldhof P, Nejsum P, Sternberg PW, Yang H, Wang Jun, Wang Jian, Gasser RB. 2011. Ascaris suum draft genome. Nature 479:529-533. doi:10.1038/nature10553

Jonassen T, Larsen PL, Clarke CF. 2001. A dietary source of coenzyme Q is essential for growth of long-lived Caenorhabditis elegans clk-1 mutants. Proc Natl Acad Sci U S A 98:421-6. doi:10.1073/pnas.021337498

Jones AK, Buckingham SD, Sattelle DB. 2005. Chemistry-to-gene screens in Caenorhabditis elegans. Nat Rev Drug Discov 4:321-330. doi:10.1038/nrd1692

Kita K. 1992. Electron-transfer complexes of mitochondria in Ascaris suum. Parasitol Today 8:155-159.

Klockiewicz M, Gaasenbeek CPH, Tielens AGM, Van Hellemond JJ, Roos MH. 2002. Rhodoquinone and Complex II of the Electron Transport Chain in Anaerobically Functioning Eukaryotes. J Biol Chem 270:31065-31070. doi:10.1074/jbc.270.52.31065

Kmetec E, Bueding E. 1961. Succinic and reduced diphosphopyridine nucleotide oxidase systems of Ascaris muscle. J Biol Chem 236:584-591.

Komuniecki R, McCrury J, Thissen J, Rubin N. 1989. Electron-transfer flavoprotein from anaerobic Ascaris suum mitochondria and its role in NADH-dependent 2-methyl branched-chain enoyl-CoA reduction. BBA - Bioenerg 975:127-131. doi:10.1016/S00052728(89)80210-5

Kotake Y. 1936. Studien über den intermediären Stoffwechsel des Tryptophans XVIII-XXIV. Hoppe-Seyler's Z Für Physiol Chem 243:237-265. doi:10.1515/bchm2.1936.243.6.237

Kurosu M, Narayanasamy P, Biswas K, Dhiman R, Crick DC. 2007. Discovery of 1,4dihydroxy-2-naphthoate [corrected] prenyltransferase inhibitors: new drug leads for multidrug-resistant gram-positive pathogens. J Med Chem 50:3973-3975. doi:10.1021/jm070638m

Kwon O, Meganathan R. 2009. Biosynthesis of Menaquinone (Vitamin K2) and Ubiquinone (Coenzyme Q). EcoSal Plus 3. doi:10.1128/ecosalplus.3.6.3.3 
Lonjers ZT, Dickson EL, Chu T-PT, Kreutz JE, Neacsu F a, Anders KR, Shepherd JN. 2012. Identification of a new gene required for the biosynthesis of rhodoquinone in Rhodospirillum rubrum. J Bacteriol 194:965-71. doi:10.1128/JB.06319-11

Ma YC, Funk M, Dunham WR, Komuniecki R. 1993. Purification and characterization of electron-transfer flavoprotein:rhodoquinone oxidoreductase from anaerobic mitochondria of the adult parasitic nematode, Ascaris suum. J Biol Chem 268:20360-20365.

Marbois BN, Clarke CF. 1996. The COQ7 Gene Encodes a Protein in Saccharomyces cerevisiae Necessary for Ubiquinone Biosynthesis. J Biol Chem 271:2995-3004. doi: 10.1074/jbc.271.6.2995

Meyerhof O. 1927. Recent investigations on the aerobic and anaerobic metabolism of carbohydrates. J Gen Physiol 8:531-542.

Minning DM, Gow AJ, Bonaventura J, Braun R, Dewhirst M, Goldberg DE, Stamler JS. 1999. Ascaris haemoglobin is a nitric oxide-activated "deoxygenase." Nature 401:497-502. doi: $10.1038 / 46822$

Mitchell P. 1975. Protonmotive redox mechanism of the cytochrome $b-c_{1}$ complex in the respiratory chain: Protonmotive ubiquinone cycle. FEBS Lett 56:1-6. doi:10.1016/00145793(75)80098-6

Mitchell P. 1961. Coupling of Phosphorylation to Electron and Hydrogen Transfer by a ChemiOsmotic type of Mechanism. Nature 191:144-148. doi:10.1038/191144a0

Momose K, Rudney H. 1972. 3-Polyprenyl-4-hydroxybenzoate synthesis in the inner membrane of mitochondria from p-hydroxybenzoate and isopentenylpyrophosphate. A demonstration of isoprenoid synthesis in rat liver mitochondria. J Biol Chem 247:39303940.

Moore HW, Folkers K. 1965. Coenzyme Q. LXII. Structure and Synthesis of Rhodoquinone, a Natural Aminoquinone of the Coenzyme Q Group. J Am Chem Soc 87:1409-1410. doi:10.1021/ja01084a065

Müller M, Mentel M, van Hellemond JJ, Henze K, Woehle C, Gould SB, Yu R-Y, van der Giezen M, Tielens AGM, Martin WF. 2012. Biochemistry and evolution of anaerobic energy metabolism in eukaryotes. Microbiol Mol Biol Rev MMBR 76:444-95. doi:10.1128/MMBR.05024-11

Nicholls P, Van Buuren KJH, Van Gelder BF. 1972. VIII. EFFECT OF CYANIDE ON THE CATALYTIC ACTIVITY. Biochim Biophys Acta 275:279-287.

Ozeir M, Pelosi L, Ismail A, Mellot-Draznieks C, Fontecave M, Pierrel F. 2015. Coq6 Is Responsible for the C4-deamination Reaction in Coenzyme Q Biosynthesis in Saccharomyces cerevisiae. J Biol Chem 290:24140-24151. doi:10.1074/jbc.M115.675744

Pettersen EF, Goddard TD, Huang CC, Couch GS, Greenblatt DM, Meng EC, Ferrin TE. 2004. UCSF Chimera--a visualization system for exploratory research and analysis. J Comput Chem 25:1605-1612. doi:10.1002/jcc. 20084

Pierrel F. 2017. Impact of Chemical Analogs of 4-Hydroxybenzoic Acid on Coenzyme Q Biosynthesis: From Inhibition to Bypass of Coenzyme Q Deficiency. Front Physiol 8:436. doi:10.3389/fphys.2017.00436

Rioux A, Komuniecki R. 1984. 2-Methylvalerate formation in mitochondria of Ascaris suum and its relationship to anaerobic energy generation. J Comp Physiol B 154:349-354.

Sali A, Blundell TL. 1993. Comparative protein modelling by satisfaction of spatial restraints. $J$ Mol Biol 234:779-815. doi:10.1006/jmbi.1993.1626 
Sangster NC, Cowling A, Woodgate RG. 2018. Ten Events That Defined Anthelmintic Resistance Research. Trends Parasitol 34:553-563. doi:10.1016/j.pt.2018.05.001

Sato M, Ozawa H. 1969. Occurrence of ubiquinone and rhodoquinone in parasitic nematodes, metastrongylus elongatus and Ascaris lumbricoides var. suis. J Biochem (Tokyo) 65:861867. doi:10.1093/oxfordjournals.jbchem.a129090

Sato M, Yamada K, Ozawa H. 1972. Rhodoquinone specificity in the reactivation of succinoxidase activity of acetone-extracted Ascaris mitochondria. Biochem Biophys Res Commun 46:578-582.

Saz HJ, Lescure OL. 1969. The functions of phosphoenolpyruvate carboxykinase and malic enzyme in the anaerobic formation of succinate by Ascaris lumbricoides. Comp Biochem Physiol 30:49-60. doi:10.1016/0010-406X(69)91296-1

Saz HJ, Vidrine A. 1959. The mechanism of formation of succinate and propionate by Ascaris lumbricoides muscle. J Biol Chem 234:2001-2005.

Sievers F, Wilm A, Dineen D, Gibson TJ, Karplus K, Li W, Lopez R, McWilliam H, Remmert M, Söding J, Thompson JD, Higgins DG. 2011. Fast, scalable generation of high-quality protein multiple sequence alignments using Clustal Omega. Mol Syst Biol 7:539. doi:10.1038/msb.2011.75

Spensley M, Del Borrello S, Pajkic D, Fraser AG. 2018. Acute Effects of Drugs on Caenorhabditis elegans Movement Reveal Complex Responses and Plasticity. G3 Bethesda Md 8:2941-2952. doi:10.1534/g3.118.200374

Stairs CW, Fawcett JP, Dellaire G, Roger AJ, Muñoz-Gómez SA, Cohen A, Eme L, Shepherd JN. 2018. Microbial eukaryotes have adapted to hypoxia by horizontal acquisitions of a gene involved in rhodoquinone biosynthesis. eLife 7. doi:10.7554/elife.34292

Stiernagle T. 2006. Maintenance of C. elegans. WormBook Online Rev C Elegans Biol 1-11. doi:10.1895/wormbook.1.101.1

Takamiya S, Matsuda M, Aoki T, Murayama K, Taka H, Matsui T. 2002. Free-Living Nematodes Caenorhabditis elegans Possess in Their Mitochondria an Additional Rhodoquinone, an Essential Component of the Eukaryotic Fumarate Reductase System. Arch Biochem Biophys 371:284-289. doi:10.1006/abbi.1999.1465

Tielens AG, Horemans AM, Dunnewijk R, van der Meer P, van den Bergh SG. 1992. The facultative anaerobic energy metabolism of Schistosoma mansoni sporocysts. $\mathrm{Mol}$ Biochem Parasitol 56:49-57.

Tielens AGM. 1994. Energy generation in parasitic helminths. Parasitol Today 10:346-352. doi:10.1016/0169-4758(94)90245-3

Tielens AGM, van den Heuvel JM, van den Bergh SG. 1984. The energy metabolism of Fasciola hepatica during its development in the final host. Mol Biochem Parasitol 13:301-307. doi:10.1016/0166-6851(84)90121-X

Trumpower BL, Houser RM, Olson RE. 1974. Studies on ubiquinone. Demonstration of the total biosynthesis of ubiquinone-9 in rat liver mitochondria. J Biol Chem 249:3041-3048.

van der Goot AT, Zhu W, Vázquez-Manrique RP, Seinstra RI, Dettmer K, Michels H, Farina F, Krijnen J, Melki R, Buijsman RC, Ruiz Silva M, Thijssen KL, Kema IP, Neri C, Oefner PJ, Nollen EAA. 2012. Delaying aging and the aging-associated decline in protein homeostasis by inhibition of tryptophan degradation. Proc Natl Acad Sci U S A 109:14912-14917. doi:10.1073/pnas.1203083109

Van Hellemond JJ, Klockiewicz M, Gaasenbeek CPH, Roos MH, Tielens AGM. 1995. Rhodoquinone and Complex II of the Electron Transport Chain in Anaerobically 
bioRxiv preprint doi: https://doi.org/10.1101/627737; this version posted May 4, 2019. The copyright holder for this preprint (which was not aCC-BY-NC 4.0 International license.

822

823

824

825

826

827

828

829

Functioning Eukaryotes. $J$ Biol Chem 270:31065-31070. doi:10.1074/jbc.270.52.31065 Van Hellemond JJ, Luijten M, Flesch FM, Gaasenbeek CPH, Tielens AGM. 1996. Rhodoquinone is synthesized de novo by Fasciola hepatica. Mol Biochem Parasitol 82:217-226. doi:10.1016/0166-6851(96)02738-7

Webb B, Sali A. 2016. Comparative Protein Structure Modeling Using MODELLER. Curr Protoc Bioinforma 54:5.6.1-5.6.37. doi:10.1002/cpbi.3

World Health Organization. 2002. Prevention and control of schistosomiasis and soil-transmitted helminthiasis: report of a WHO expert committee. 
a.

Aerobic

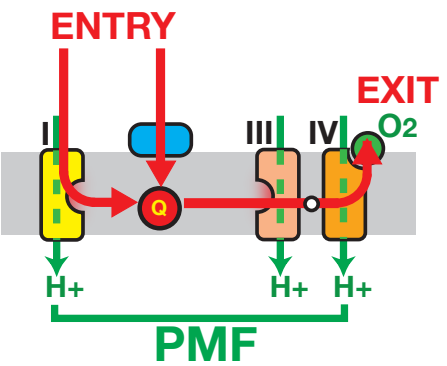

c.

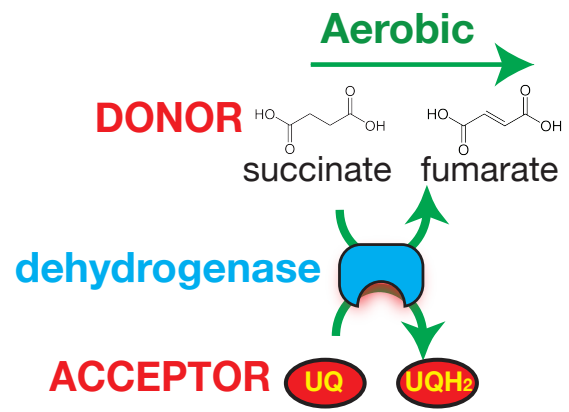

d.<smiles>COC1=C(OC)C(OC)C([N+](=O)[O-])=C(C=O)C1=O</smiles>

b. Anaerobic/ETC block

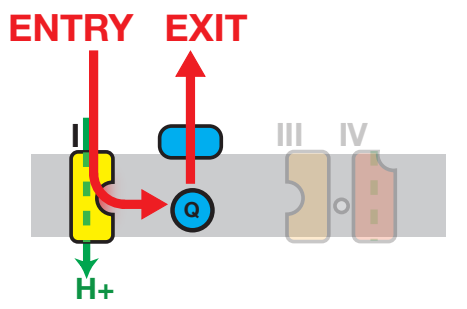

PMF
Anaerobic/ETC block

succinate fumarate

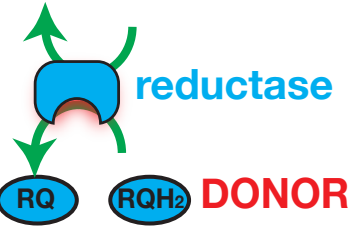<smiles>COC1=C(N)C(=O)C([N+](=O)[O-])=C(C)C1=O</smiles> 
bioRxiv preprint doi: https://doi.org/10.1101/627737; this version posted May 4,2019 . The copyright holder for this preprint (which was not certified by peer review) is the author/funder, who has granted bioRxiv a license to display the preprint in perpetuity. It is made available under aCC-BY-NC 4.0 International license.

a.

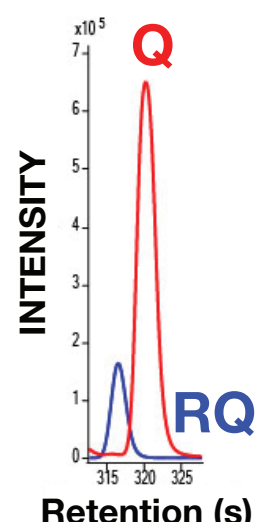

b.

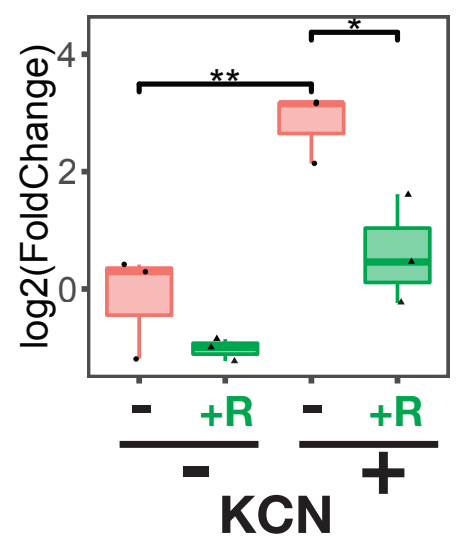



aCC-BY-NC 4.0 International license.

a.

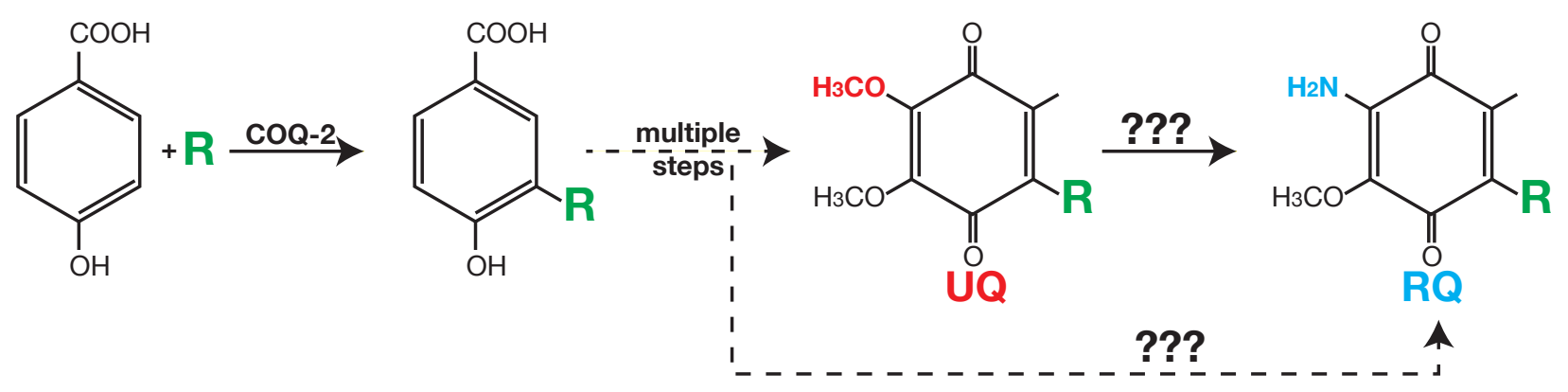<smiles>[R]c1ccc(N)c(C(=O)OCC(=O)O)c1</smiles><smiles>CC1=C(C)C(C)=C(N)C(=O)C1=O</smiles>

RQ

b.

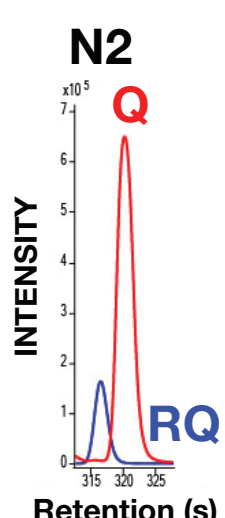

c.
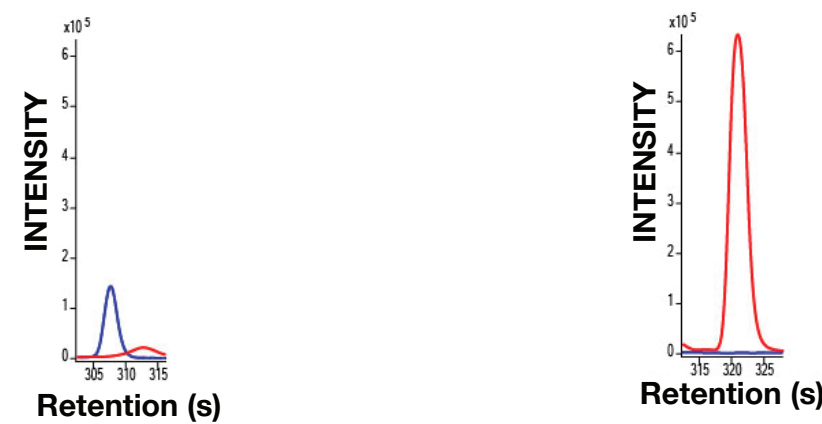

Retention (s) 
a.<smiles>N[C@@H](Cc1c[nH]c2ccccc12)C(=O)O</smiles>

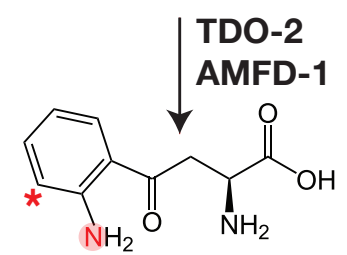

Kynurenine<smiles>CC1=C(O)C(=O)C(Oc2ccccc2)=C(N)C1=O</smiles>

b.

$-{ }^{14} \mathrm{~N}$ Trp

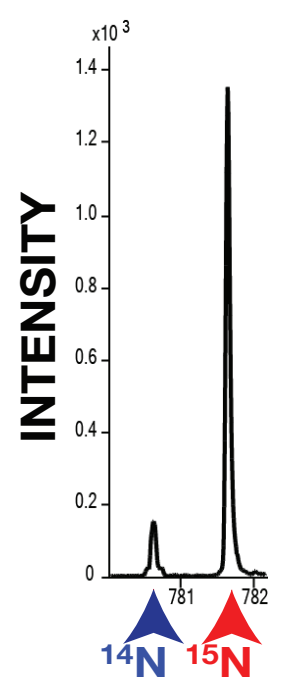

$+{ }^{14} \mathbf{N}$ Trp

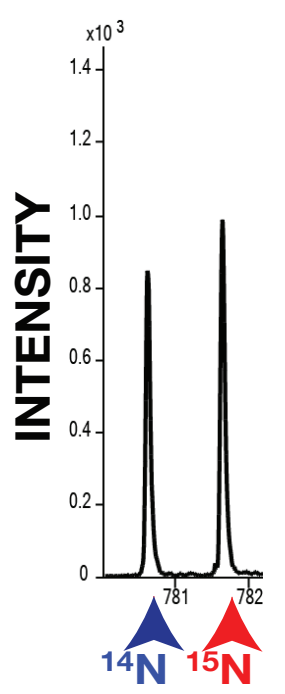


bioRxiv preprint doi: https://doi.org/10.1101/627737; this version posted May 4, 2019. The copyright holder for this preprint (which was not certified by peer review) is the author/funder, who has granted bioRxiv a license to display the preprint in perpetuity. It is made available under aCC-BY-NC 4.0 International license.

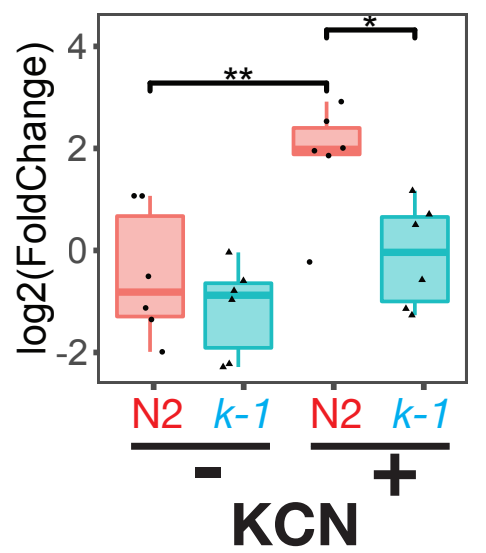


a.
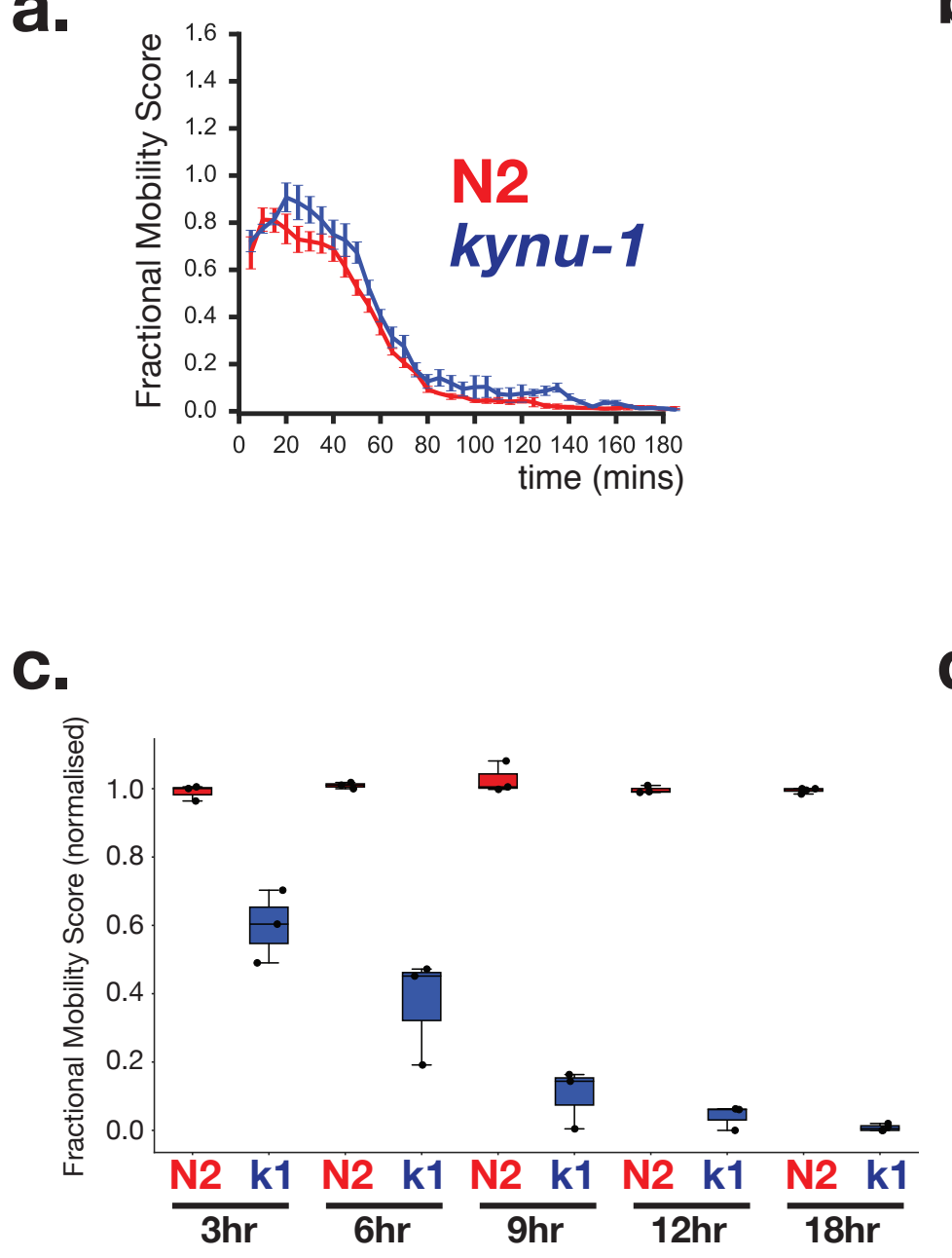

b.

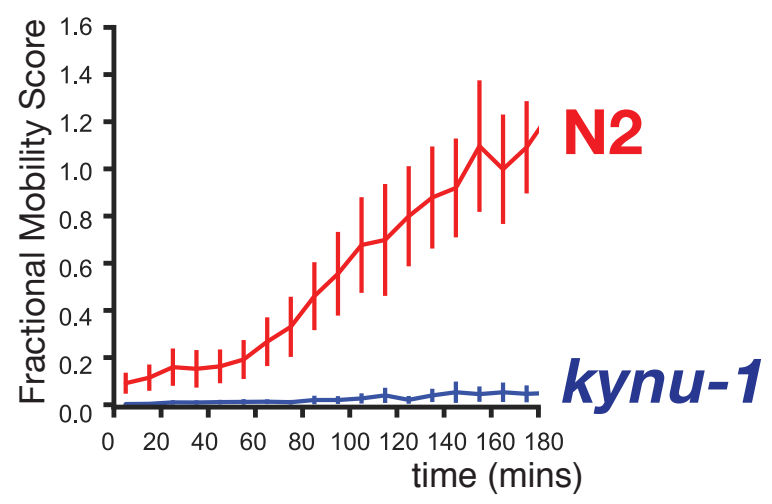

d.

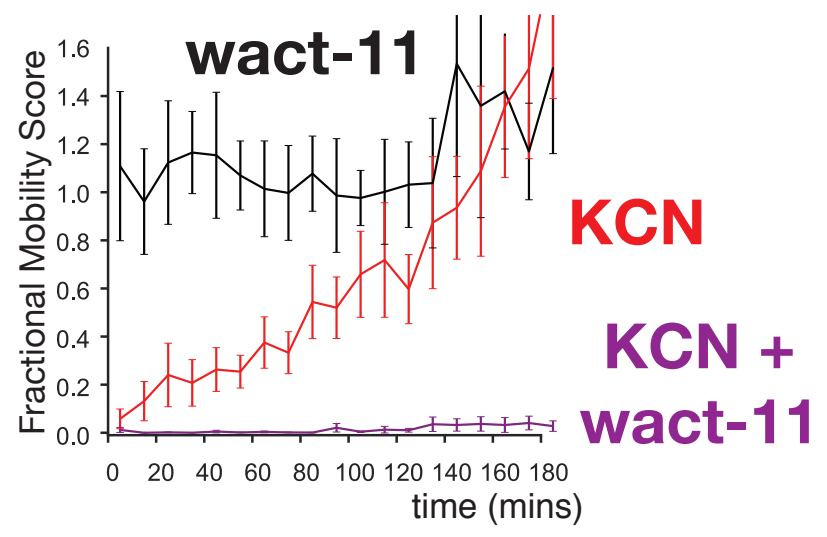


bioRxiv preprint doi: https://doi.org/10.1101/627737; this version posted May 4, 2019. The copyright holder for this preprint (which was not certified by peer review) is the author/funder, who has granted bioRxiv a license to display the preprint in perpetuity. It is made available under aCC-BY-NC 4.0 International license.
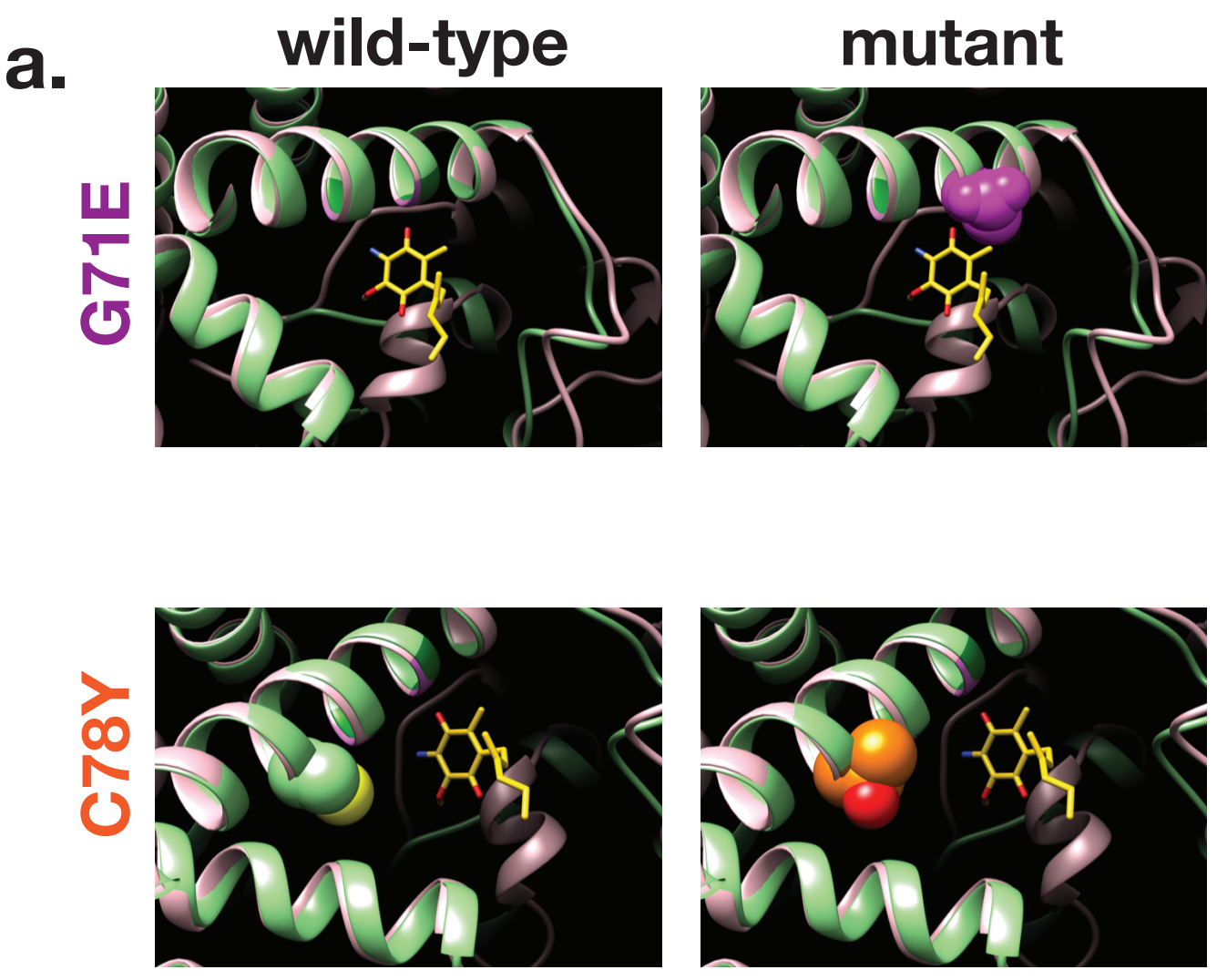

b.

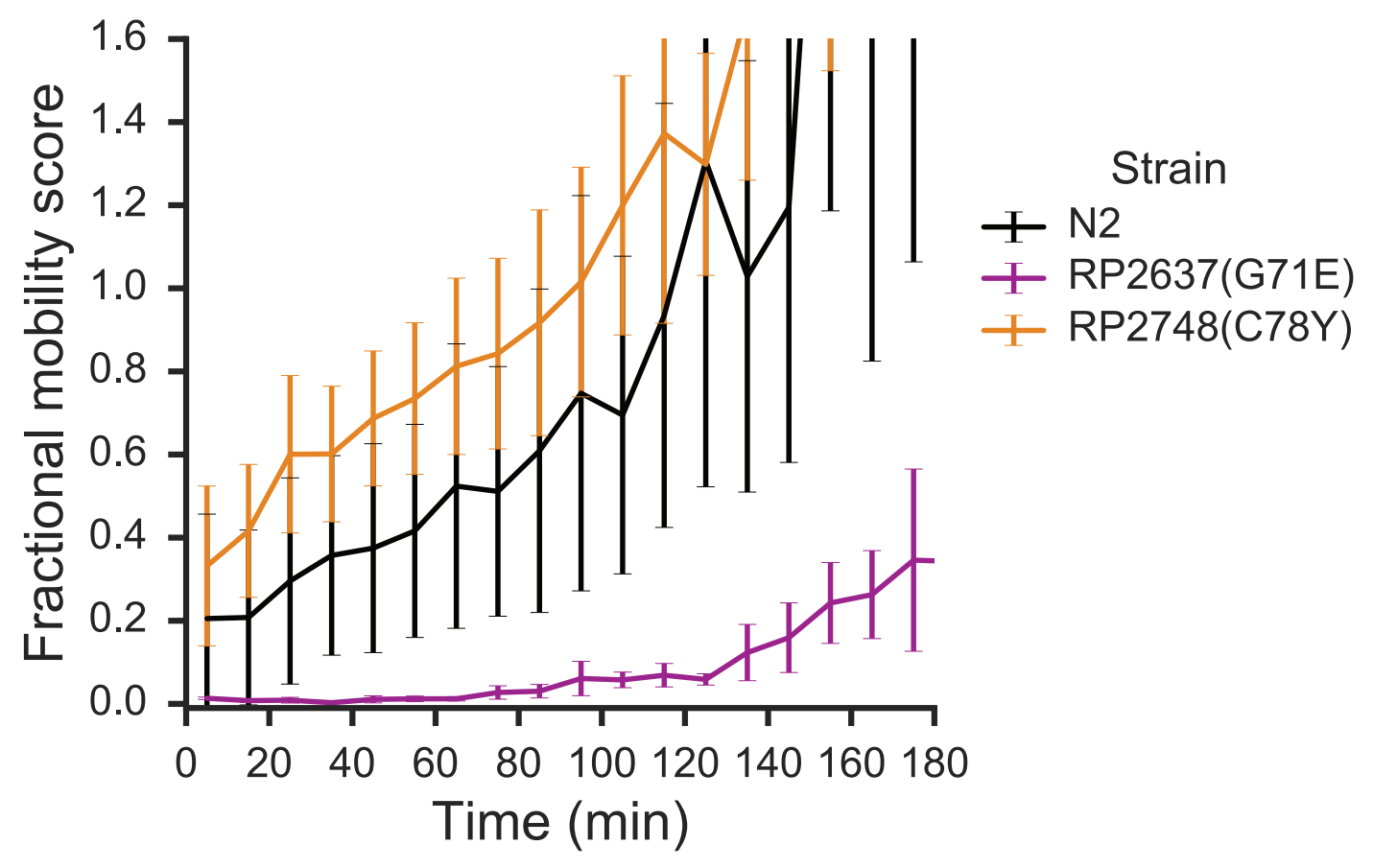


a.

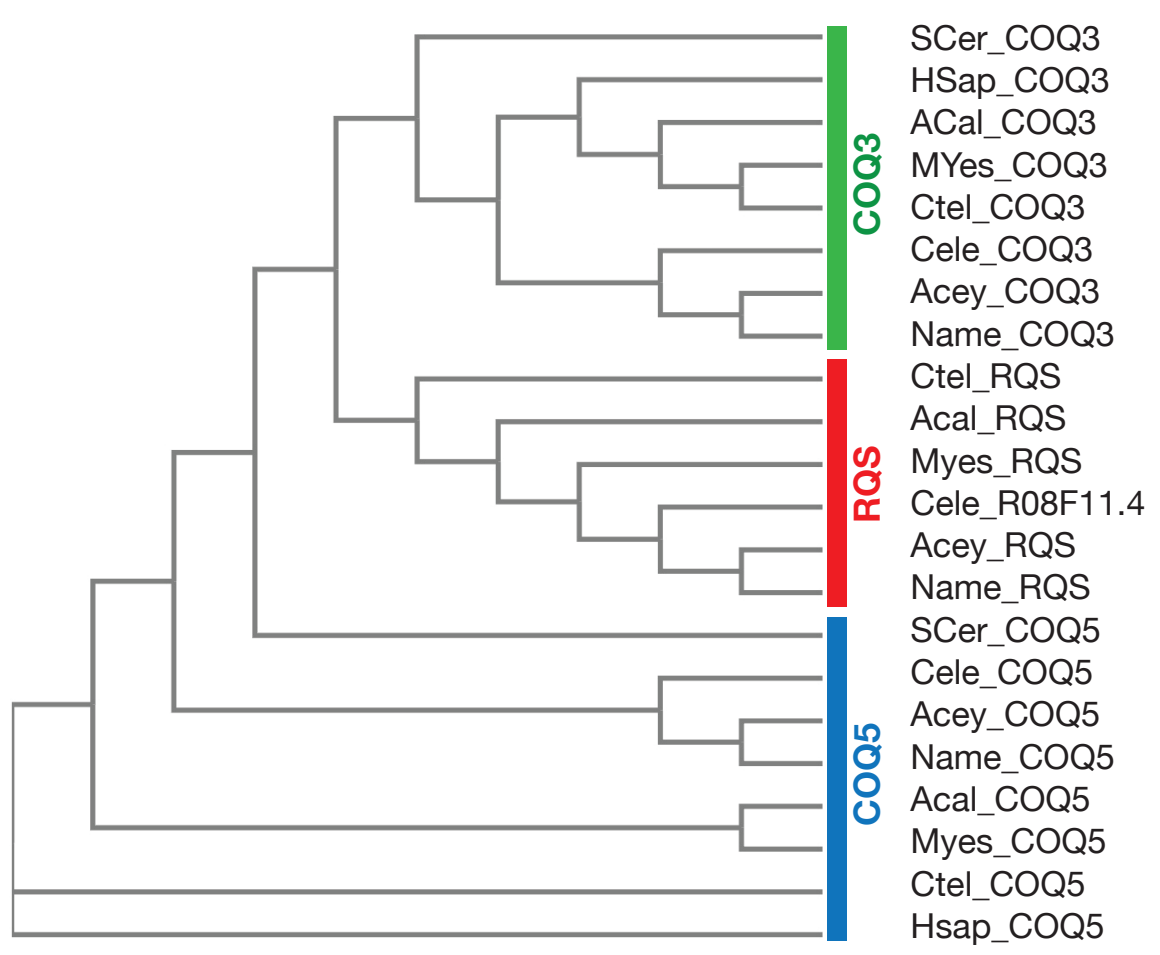

b.

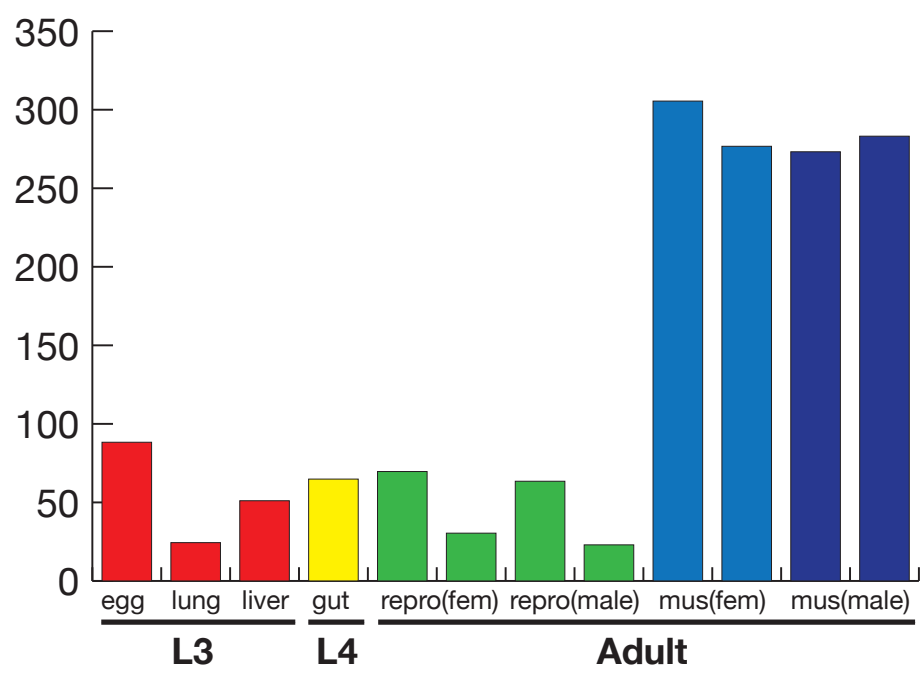



aCC-BY-NC 4.0 International license.

a.

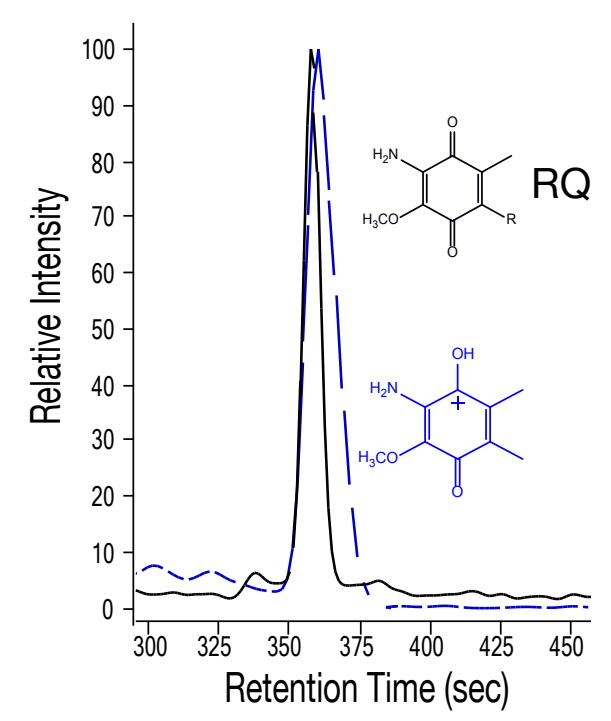

c.
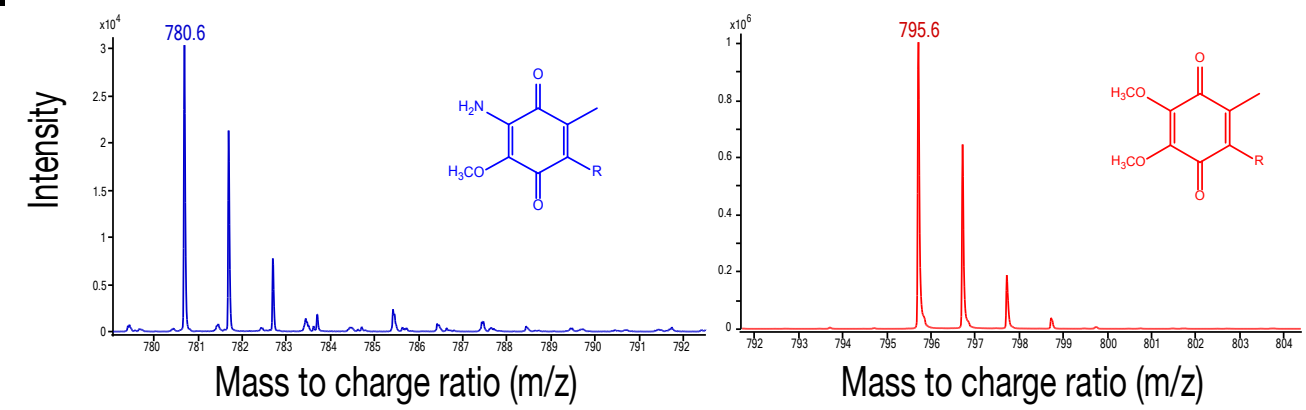

d.

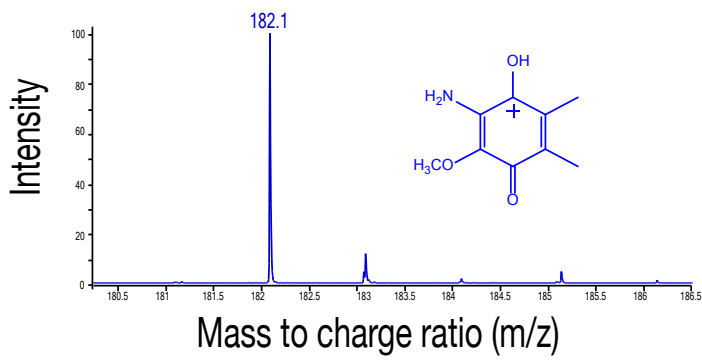

b.

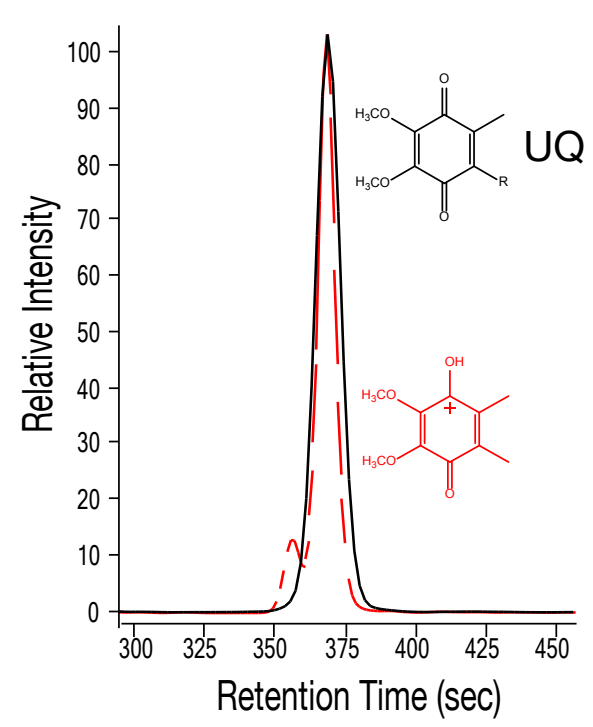

